\title{
RIGHT ANGLED ARTIN GROUPS AND PARTIAL COMMUTATION, OLD AND NEW
}

\author{
LAURENT BARTHOLDI, HENRIKA HÄRER, AND THOMAS SCHICK
}

\begin{abstract}
We systematically treat algebraic objects with free partially commuting generators and give short and modern proofs of the various relations between them. These objects include right angled Artin groups, polynomial rings, Lie algebras, and restricted Lie algebras in partially commuting free generators. In particular, we compute the $p$-central and exponent- $p$ series of all right angled Artin groups, and compute the dimensions of their subquotients. We also describe their associated Lie algebras, and relate them to the cohomology ring of the group as well as to polynomial and power series rings in partially commuting variables. We finally show how the growth series of these various objects are related to each other.
\end{abstract}

\section{INTRODUCTION}

Right angled Artin groups (RAAGs) are a prominent geometric/combinatorial class of groups. Originally introduced as "partially commuting free groups", they interpolate in an interesting way between free groups and free abelian groups. Of particular interest are several additional algebraic objects which are canonically coming along and are closely related to the structure of the RAAGs, in particular (graded) Lie algebras and polynomial rings, both in free partially commuting generators. The purpose of this article is to give a complete description of many relevant properties and relations, offering modern and accessible proofs. Many of the results quoted below appear already in other sources, though the computation of the exponent- $p$ and lower $p$-central series is new.

1.1. The actors. Let $\Gamma$ be an undirected graph, with vertex set $V$ and edge set $E$ (consisting of 2-element subsets of $V$ ). The right angled Artin group (RAAG) $A_{\Gamma}$ associated with $\Gamma$ is the group defined in terms of generators and relations as

$$
\left.A_{\Gamma}=\langle V| v w=w v \text { whenever }\{v, w\} \in E\right\rangle .
$$

The purpose of this note is to describe classical subgroup series in $A_{\Gamma}$ such as the lower-central and $p$-lower-central series, and relate them to other algebraic objects defined in terms of $\Gamma$ as follows.

Let $\mathbf{k}$ be a commutative ring. We define unital associative $\mathbf{k}$-algebras

$$
\begin{aligned}
& \left.R_{\Gamma}=\langle V| v w=w v \text { whenever }\{v, w\} \in E\right\rangle, \\
& \left.S_{\Gamma}=\langle V| v^{2}=0, v w=-w v \forall v, w \in V, \text { and } v w=0 \text { whenever }\{v, w\} \notin E\right\rangle .
\end{aligned}
$$

Note that $R_{\Gamma}$ is the familiar algebra of polynomials in partially commuting variables, and similarly $S_{\Gamma}$ can be considered as an exterior algebra in partially commuting variables.

Observe that $R_{\Gamma}$ and $S_{\Gamma}$ are graded algebras with $\operatorname{deg}(v)=1$ for all $v \in V$. Therefore, they admit a natural topology, in which basic neighbourhoods of 0 (say in $R_{\Gamma}$ ) are spans of the set of all monomials of degree $\geq n$. We define

$$
\overline{R_{\Gamma}}=\text { the completion of } R_{\Gamma} \text { in this topology. }
$$

Just as $R_{\Gamma}$ is a non-commutative polynomial algebra, $\overline{R_{\Gamma}}$ is an algebra of power series in partially commuting variables.

Date: May 1, 2020.

Part of this work is contained in the bachelor thesis 34 of Henrika Härer, née Runde. 
We also define a Lie algebra over $\mathbf{k}$,

$$
\left.L_{\Gamma}=\langle V|[v, w]=0 \text { whenever }\{v, w\} \in E\right\rangle,
$$

and, if $\mathbf{k}$ is an algebra over $\mathbb{F}_{p}$, a restricted Lie algebra (see Section 2 for a review of restricted Lie algebras)

$$
\left.L_{\Gamma, p}=\langle V|[v, w]=0 \text { whenever }\{v, w\} \in E\right\rangle_{p} .
$$

Let us have a look at the extreme cases.

(1) If $\Gamma$ is the complete graph on $d$ vertices then $A_{\Gamma} \cong \mathbb{Z}^{d}, R_{\Gamma}$ is the polynomial algebra in $d$ variables $\mathbf{k}\left[X_{1}, \ldots, X_{d}\right], S_{\Gamma}$ is the Grassmann algebra $\bigwedge^{*}\left(\mathbf{k}^{d}\right)$, and $L_{\Gamma} \cong \mathbf{k}^{d}$ with trivial bracket.

(2) If $\Gamma$ is the empty graph on $d$ vertices then $A_{\Gamma}$ is the free group $F_{d}, R_{\Gamma}$ is the free associative algebra on $d$ generators, $S_{\Gamma} \cong \mathbf{k} \cdot 1 \oplus \mathbf{k}^{d}$ with trivial multiplication except $1 \cdot x=x$, and $L_{\Gamma}$ is the free Lie algebra on $d$ generators; for more details see Section 1.4

1.2. Subgroup series. Let $G$ be any discrete group, and let $\rho: G \rightarrow R^{\times}$be a representation of $G$ in an associative augmented k-algebra $R$ with augmentation ideal $\varpi$ (namely, an algebra equipped with an epimorphism to $\mathbf{k}$ with kernel $\varpi$ ). With this representation is associated a natural sequence of subgroups, called generalized dimension subgroups,

$$
\delta_{n, \rho}:=\rho^{-1}\left(1+\varpi^{n}\right)=\operatorname{ker}\left(G \rightarrow\left(R / \varpi^{n}\right)^{\times}\right) .
$$

In case $R=\mathbf{k} G$ and $\rho$ is the regular representation, we write $\delta_{n, \mathbf{k} G}$ for $\delta_{n, \rho}$.

In addition, there are classical subgroup series, defined intrinsically within $G$ :

- the lower central series $\left(\gamma_{n}\right)$ given by $\gamma_{1}=G$ and $\gamma_{n}=\left[\gamma_{n-1}, G\right]$;

- the rational lower central series $\gamma_{n, 0}=\left\{g \in G \mid g^{k} \in \gamma_{n}\right.$ for some $\left.k \neq 0\right\}$;

- for a prime $p$ fixed throughout the discussion, the exponent- $p$ central series $\lambda_{n, p}$ given by $\lambda_{1, p}=G$ and $\lambda_{n, p}=\left[\lambda_{n-1, p}, G\right] \lambda_{n-1, p}^{p}$, or more directly $\lambda_{n, p}=\prod_{m+i>n} \gamma_{m}^{p^{i}}$

- again for a prime $p$ fixed throughout the discussion, the Brauer-JenningsLazard-Zassenhaus series [18,22, 42, also called p-dimension or p-central series, given by $\gamma_{1, p}=G$ and $\gamma_{n, p}=\left[\gamma_{n-1, p}, G\right] \gamma_{\lceil n / p\rceil, p}^{p}$, or more directly $\gamma_{n, p}=\prod_{m p^{i} \geq n} \gamma_{m}^{p^{i}}$.

All these series are central, meaning that $\gamma_{n} / \gamma_{n+1}$ belongs to the center of $G / \gamma_{n+1}$, etc. We moreover have $\left[\gamma_{m}, \gamma_{n}\right] \subseteq \gamma_{m+n}$, etc. A classical consequence [25, Section 5.3] is that $\bigoplus_{n \geq 1} \gamma_{n} / \gamma_{n+1}$, etc., are graded Lie algebras over $\mathbb{Z}$. The addition is induced by the group multiplication and the Lie bracket is induced by the commutator.

The groups $\gamma_{n, 0}$ enjoys the extra property that $\gamma_{n, 0} / \gamma_{n+1,0}$ is torsion-free (and it is the fastest descending central series with this property), so $\bigoplus_{n \geq 1} \gamma_{n, 0} / \gamma_{n+1,0}$ is $\mathbb{Z}$-free. In particular, if $\gamma_{n} / \gamma_{n+1}$ is torsion free for each $n$, then $\gamma_{n, 0}=\gamma_{n}$ for each $n$.

We have $\lambda_{n, p}^{p} \subseteq \lambda_{n+1, p}$ so $\bigoplus_{n>1} \lambda_{n, p} / \lambda_{n+1, p}$ is an elementary abelian $p$-group. Similarly, $\gamma_{n, p}^{p} \subseteq \gamma_{n p, p}$. Furthermore, these series are fastest descending under these requirements. It is now classical [42] that $\bigoplus_{n \geq 1} \gamma_{n, p} / \gamma_{n+1, p}$ is a restricted Lie algebra over $\mathbb{F}_{p}$. The additional, "p-power" operation as part of the restricted Lie algebra structure is induced by the $p$-power operation in the group.

Classical results identify $\delta_{n, \mathbf{k} G}$ with some of the above series in case $\mathbf{k}$ is a field: we have $\delta_{n, \mathbf{k} G}=\gamma_{n, p}$ where $p \geq 0$ is the characteristic of $\mathbf{k}$ [15, 18, 19. However, for general $G$, the identification of $\delta_{n, \mathbb{Z} G}$ is a fundamental open problem of group theory. 
1.3. Results. We consider the series defined above for the group $A_{\Gamma}$. The main purpose of this text is to exhibit numerous relations between these algebraic objects; detailed definitions and proofs will be given in subsequent sections. The main tool is an extension to $A_{\Gamma}$ of Magnus's work on the free group [25, Section 5], embedding it into the units of the free non-commuting power series ring. This extension seems first considered in 7 .

Recall that a commutative ring $\mathbf{k}$ is fixed. Denote by $\varpi$ the augmentation ideal of $R_{\Gamma}$ (i.e. the ideal of polynomials in partially commuting variables with zero constant term), and by $\varpi\left(A_{\Gamma}\right)$ the augmentation ideal of $\mathbf{k} A_{\Gamma}$.

Theorem 1.1 (Augmentation ideals). For all $n$ we have

$$
\varpi\left(A_{\Gamma}\right)^{n} / \varpi\left(A_{\Gamma}\right)^{n+1} \cong \varpi^{n} / \varpi^{n+1} .
$$

We remind the reader that Koszul algebras are a particular kind of associative algebras (see 31] or Section 4) for which a "small" projective resolution may easily be computed. Moreover, there is the important concept of Koszul duality. We obtain the following results, which for $\mathbf{k}=\mathbb{Q}$ already appear in [30].

Theorem 1.2 (Group cohomology). Let $S^{1}$ be the circle with base point $*$. The following subspace of the torus $\left(S^{1}\right)^{V}$ is a classifying space for $A_{\Gamma}$ :

$$
X_{\Gamma}=\bigcup_{C \subseteq V \text { a clique }}\left(S^{1}\right)^{C} \times\{*\}^{V \backslash C} .
$$

We have $H^{*}\left(A_{\Gamma} ; \mathbf{k}\right)=S_{\Gamma}$.

The rings $R_{\Gamma}$ and $S_{\Gamma}$ are Koszul algebras, and Koszul duals to each other: $\left(S_{\Gamma}\right)^{!}=R_{\Gamma}$.

Theorem 1.3i (Central series and dimension subgroups). We have

$$
\gamma_{n}=\gamma_{n, 0} \quad \text { and } \quad \bigcap_{n \in \mathbb{Z}} \gamma_{n, 0}=\bigcap_{n \in \mathbb{Z}} \gamma_{n, p}=\{1\} .
$$

In particular, $A_{\Gamma}$ is finitely generated and residually torsion-free nilpotent, so (by 13 , Theorem 2.1]) $A_{\Gamma}$ is also a residually finite p-group for every $p$.

Theorem 1.3ii (Central series and dimension subgroups). There is a faithful representation

$$
\mu: A_{\Gamma} \rightarrow{\overline{R_{\Gamma}}}^{\times} ; \quad v \mapsto 1+v \quad \text { for } v \in V .
$$

The corresponding generalized dimension subgroups satisfy

$$
\delta_{n, \mu}= \begin{cases}\gamma_{n, 0} & \text { if } \mathbf{k} \text { has characteristic } 0 \\ \gamma_{n, p} & \text { if } \mathbf{k} \text { has characteristic } p\end{cases}
$$

Together with Theorem 1.1 we obtain an isomorphism of filtered associativ 1 kalgebras

$$
\overline{\mathbf{k} A_{\Gamma}}:=\lim \left(\mathbf{k} A_{\Gamma} / \varpi(G)^{n}\right) \stackrel{\cong}{\rightrightarrows} \lim \left(R_{\Gamma} / \varpi^{n}\right)=\overline{R_{\Gamma}} .
$$

In particular, the classical dimension subgroups coincide:

$$
\delta_{n, \mathbf{k} A_{\Gamma}}=\delta_{n, \mu}
$$

The Lie algebras $L_{\Gamma}$ and $L_{\Gamma, p}$ are tightly connected to their associative counterparts:

Theorem 1.4i (Lie algebras). The algebra $R_{\Gamma}$ is a Hopf algebra. If the ring $\mathbf{k}$ is $a \mathbb{Z}$-free module then we have

$$
L_{\Gamma} \cong \operatorname{Primitives}\left(R_{\Gamma}\right) \text { and } R_{\Gamma} \cong U\left(L_{\Gamma}\right),
$$

the universal enveloping algebra of $L_{\Gamma}$, while if $\mathbf{k}$ is an $\mathbb{F}_{p}$-algebra then

$$
L_{\Gamma, p} \cong \operatorname{Primitives}\left(R_{\Gamma}\right) \text { and } R_{\Gamma} \cong U_{p}\left(L_{\Gamma, p}\right),
$$

\footnotetext{
${ }^{1}$ but not Hopf algebras; see Theorem 1.6 below!
} 
the p-universal enveloping algebra of $L_{\Gamma, p}$. The Lie algebra cohomology of $L_{\Gamma}$ is

$$
H^{*}\left(L_{\Gamma} ; \mathbf{k}\right) \cong S_{\Gamma} .
$$

All the above isomorphisms are natural, in the sense that they are induced by the identity map $V \rightarrow V$, and therefore compatible with homomorphisms induced by a map of graphs $V \rightarrow V^{\prime}$.

The Lie algebra associated with the lower central series was already determined in [8] as $L_{\Gamma}$. We extend this result as follows:

Theorem 1.4ii (Lie algebras). For any ring $\mathbf{k}$, we have, as Lie algebras,

$$
L_{\Gamma} \cong \bigoplus_{n \geq 1}\left(\gamma_{n} / \gamma_{n+1}\right) \otimes_{\mathbb{Z}} \mathbf{k}
$$

If $\mathbb{F}_{p} \subseteq \mathbf{k}$ then as restricted Lie algebras

$$
L_{\Gamma, p} \cong \bigoplus_{n \geq 1}\left(\gamma_{n, p} / \gamma_{n+1, p}\right) \otimes_{\mathbb{Z}} \mathbf{k} .
$$

If $\mathbb{F}_{p} \subseteq \mathbf{k}$ and $p \geq 3$ then with $\mathbf{k}[\pi]$ the polynomial ring in one degree-1 variable $\pi$

$$
L_{\Gamma} \otimes_{\mathbf{k}} \mathbf{k}[\pi] \cong \bigoplus_{n \geq 1}\left(\lambda_{n, p} / \lambda_{n+1, p}\right) \otimes_{\mathbb{Z}} \mathbf{k}
$$

under that isomorphism, multiplication by $\pi$ corresponds to the map induced by $\lambda_{n, p} \ni g \mapsto g^{p} \in \lambda_{n+1, p}$.

All the above isomorphisms are natural, in the sense that they are induced by the identity map $V \rightarrow V$, and therefore compatible with homomorphisms induced by a map of graphs $V \rightarrow V^{\prime}$.

For a graded algebra $R=\bigoplus_{n \geq 0} R_{n}$ over $\mathbf{k}$ such that each $R_{n}$ is a finitely generated free $\mathbf{k}$-module, recall that its Poincaré series is the power series

$$
\Phi_{R}(t)=\sum_{n \geq 0} \operatorname{rank}\left(R_{n}\right) t^{n}
$$

For a group $G=\langle X\rangle$, its growth series is $\Phi_{G}(t)=\sum_{g \in G} t^{|g|}$, with $|g|$ denoting the word length of $g \in G$ (word length and growth series depend on the fixed generating set $X)$. The first two claims of the following result appear in [9]:

Theorem 1.5 (Poincaré and growth series). The Poincaré series of $S_{\Gamma}$ is

$$
\Phi_{S_{\Gamma}}(t)=\sum_{n=0}^{|V|} c_{n}(\Gamma) t^{n}
$$

where $c_{n}(\Gamma)$ denotes the number of cliques of size $n$ (i.e. complete subgraphs of $\Gamma$ with $n$ vertices).

The Poincaré series of $R_{\Gamma}$ and $S_{\Gamma}$ are connected by the relation

$$
\Phi_{R_{\Gamma}}(t) \cdot \Phi_{S_{\Gamma}}(-t)=1
$$

and the growth series of $A_{\Gamma}$ is

$$
\Phi_{A_{\Gamma}}(t)=\Phi_{R_{\Gamma}}\left(\frac{2 t}{1+t}\right) .
$$

In our next result, originally appearing in [20, Theorem 16.10], we determine the Malcev completion of $A_{\Gamma}$. We refer to [26, 33] and the more recent [29] for a review of this construction.

Theorem 1.6 (Malcev completions). Assume $\mathbf{k}=\mathbb{Q}$. There is then an isomorphism $\mu_{\exp }: \overline{R_{\Gamma}} \rightarrow \overline{\mathbb{Q} A_{\Gamma}}$ of filtered, complete Hopf algebras; via this isomorphism, $\overline{L_{\Gamma}}$ is the Malcev Lie algebra of $A_{\Gamma}$, and the Malcev completion of $A_{\Gamma}$ is given on generators by

$$
A_{\Gamma} \rightarrow \exp \left(L_{\Gamma}\right) \subset \overline{R_{\Gamma}} \text { via the classical power series } v \mapsto \sum_{n \geq 0} \frac{v^{n}}{n !} \forall v \in V \text {. }
$$


We also show the following related result on formality in the sense of rational homotopy theory; see Section 8 for a review of the notion.

Theorem 1.7 (Formality). The classifying space $X_{\Gamma}$ of $A_{\Gamma}$ of (11) is formal.

1.4. Examples and illustrations. Let us consider, as sketched in the Introduction, the two extreme cases of graphs $\Gamma$, the complete and empty graphs.

If $\Gamma$ is the complete graph on $V$, then $A_{\Gamma}$ is free Abelian with basis $V$, and $R_{\Gamma}$ is a usual polynomial algebra in variables $V$. The standard Koszul complex is given by the exterior algebra $S_{\Gamma}=\bigwedge^{*}(V)$, and coincides with the cohomology ring of $A_{\Gamma}=\mathbb{Z} V$. The classifying space $X_{\Gamma}$ is the usual torus $\left(S^{1}\right)^{V}$. The exponent- $p$ central series satisfies $\lambda_{n, p}=p^{n-1} \mathbb{Z} V$, and the $p$-dimension series satisfies $\gamma_{n, p}=$ $p^{i} \mathbb{Z} V$ whenever $p^{i-1}<n \leq p^{i}$. The growth series are readily computed as

$$
\Phi_{A_{\Gamma}}(t)=\left(\frac{1+t}{1-t}\right)^{|V|}, \quad \Phi_{R_{\Gamma}}(t)=\left(\frac{1}{1-t}\right)^{|V|}, \quad \Phi_{S_{\Gamma}}(t)=(1+t)^{|V|} .
$$

If, on the other hand, $\Gamma$ is the empty graph on $V$, then $A_{\Gamma}$ is free with basis $V$, and $R_{\Gamma}$ is a polynomial algebra in non-commuting variables $V$. The algebra $S_{\Gamma}$ is reduced to $\mathbf{k} \oplus \mathbf{k} V$ with $V^{2}=0$, and coincides with the cohomology ring of $A_{\Gamma}$. The classifying space $X_{\Gamma}$ is a wedge of $|V|$ circles. The Lie algebras $L_{\Gamma}$ and the restricted Lie algebra $L_{\Gamma, p}$ are free. The growth series are readily computed as

$$
\Phi_{A_{\Gamma}}(t)=\frac{1+t}{1-(2|V|-1) t}, \quad \Phi_{R_{\Gamma}}(t)=\frac{1}{1-|V| t}, \quad \Phi_{S_{\Gamma}}(t)=1+|V| t .
$$

These results can be seen as special cases of the following constructions. If $\Gamma$ is the disjoint union of two graphs $\Gamma_{1} \sqcup \Gamma_{2}$, then $A_{\Gamma}=A_{\Gamma_{1}} * A_{\Gamma_{2}}$ is a free product of groups, and similarly $L_{\Gamma}$ and $R_{\Gamma}$ are free products in their respective categories, and $S_{\Gamma}=S_{\Gamma_{1}} \oplus S_{\Gamma_{2}} /(1 \oplus 0=0 \oplus 1)$. The space $X_{\Gamma}$ is the wedge (one-point union) of $X_{\Gamma_{1}}$ and $X_{\Gamma_{2}}$, and the growth series of $A_{\Gamma}, R_{\Gamma}, S_{\Gamma}$ may be easily be deduced from those of $A_{\Gamma_{1}}, A_{\Gamma_{2}}$, etc.:

$$
\begin{aligned}
& 1-\frac{1}{\Phi_{A_{\Gamma}}}=\left(1-\frac{1}{\Phi_{A_{\Gamma_{1}}}}\right)+\left(1-\frac{1}{\Phi_{A_{\Gamma_{2}}}}\right), \\
& 1-\frac{1}{\Phi_{R_{\Gamma}}}=\left(1-\frac{1}{\Phi_{R_{\Gamma_{1}}}}\right)+\left(1-\frac{1}{\Phi_{R_{\Gamma_{2}}}}\right), \\
& 1-\Phi_{S_{\Gamma}}=\left(1-\Phi_{S_{\Gamma_{1}}}\right)+\left(1-\Phi_{A_{\Gamma_{2}}}\right) .
\end{aligned}
$$

If $\Gamma$ is the join of two graphs $\Gamma_{1}$ and $\Gamma_{2}$, namely the graph obtained from $\Gamma_{1} \sqcup \Gamma_{2}$ by adding all edges between $\Gamma_{1}$ and $\Gamma_{2}$, then $A_{\Gamma}=A_{\Gamma_{1}} \times A_{\Gamma_{2}}$ is a direct product, and similarly $L_{\Gamma}=L_{\Gamma_{1}} \times L_{\Gamma_{2}}$ and $R_{\Gamma}=R_{\Gamma_{1}} \otimes R_{\Gamma_{2}}$, while $S_{\Gamma}$ is $S_{\Gamma_{1}} \otimes S_{\Gamma_{2}}$ qua k-module, with product $(a \otimes b)(c \otimes d)=(-1)^{\operatorname{deg}(b) \operatorname{deg}(c)}(a c \otimes b d)$. The classifying space is $X_{\Gamma}=X_{\Gamma_{1}} \times X_{\Gamma_{2}}$, and the growth series $\Phi_{A_{\Gamma}}, \Phi_{R_{\Gamma}}$ and $\Phi_{S_{\Gamma}}$ behave multiplicatively:

$$
\Phi_{A_{\Gamma}}=\Phi_{A_{\Gamma_{1}}} \cdot \Phi_{A_{\Gamma_{2}}}, \quad \Phi_{R_{\Gamma}}=\Phi_{R_{\Gamma_{1}}} \cdot \Phi_{R_{\Gamma_{2}}}, \quad \Phi_{S_{\Gamma}}=\Phi_{S_{\Gamma_{1}}} \cdot \Phi_{S_{\Gamma_{2}}} .
$$

Finally, all the objects constructed are functorial, in the sense that graph morphisms induce maps between the corresponding objects: if $\Gamma, \Gamma^{\prime}$ are graphs and $f: \Gamma \rightarrow \Gamma^{\prime}$ is a map from the vertex set of $\Gamma$ to that of $\Gamma^{\prime}$ sending edges of $\Gamma$ to edges of $\Gamma^{\prime}$, then there is an induced group homomorphism $f_{*}: A_{\Gamma} \rightarrow A_{\Gamma^{\prime}}$, ring homomorphism $R_{\Gamma} \rightarrow R_{\Gamma^{\prime}}$ and $S_{\Gamma^{\prime}} \rightarrow S_{\Gamma}$ (note the direction!), etc. Furthermore, if $f$ is injective and full (meaning that $\{f(v), f(w)\}$ is an edge in $\Gamma^{\prime}$ precisely when $\{v, w\}$ is an edge in $\Gamma$ ) then the corresponding group and ring homomorphisms are injective.

1.5. Structure of the article. The article introduces and relies on quite a number of different concepts (Hopf algebras, the Magnus map, ... ). These are introduced one after the other in the following sections. In particular, Section 2 collects some basic information about (restricted) Lie algebras and Hopf algebras which we use as technical tools; we prove the first part of Theorem 1.4i in it. 
Section 3 introduces the Magnus map, which embeds the group $A_{\Gamma}$ into the units of the partially commuting power series ring $\overline{R_{\Gamma}}$. We show that this map is compatible with the central series filtrations (and dimension series filtrations). The explicit knowledge of the structure of the power series ring can be transferred to $A_{\Gamma}$ to give the desired information about the latter. We also prove Theorem 1.1 in it.

We next introduce cohomological notions in Section 4, and use them to prove Theorem 1.2 .

We study central series in more depth in Section 5 , and prove there the first, easy part of Theorem 1.3i. The second part requires more knowledge on the Lie algebras $L_{\Gamma}$, which we describe in Section 6, we prove Theorems 1.3ii and 1.4ii there. We also complete there the proof of Theorem 1.4i that pertains to Lie algebra cohomology.

Finally Section 7 proves Theorem 1.5 and Section 8 proves Theorem 1.6 . We apologize to the reader if the proofs are not given in strictly linear order; we found it preferable to prove individual statements of the main results where the appropriate tools were introduced.

\section{LIE AND HOPF ALGEBRAS}

We first recall from [17 that a restricted Lie algebra over $\mathbf{k}$, in characteristic $p$, is a Lie algebra equipped with an extra operation, written $x \mapsto x^{[p]}$, called the p-mapping and subject to the following axioms, where we use the standard multicommutator convention $[x, y, z]=[x,[y, z]]$, etc. For all $x, y$ in the Lie algebra and $\alpha \in \mathbf{k}$,

$$
\begin{gathered}
{\left[y, x^{[p]}\right]=[y, x, \ldots, x] \quad(p \text { factors ' } x \text { ' }) ; \quad(\alpha x)^{[p]}=\alpha^{p} x^{[p]} ;} \\
(x+y)^{[p]}=x^{[p]}+y^{[p]}+\sum_{i=1}^{p-1} s_{i}(x, y)
\end{gathered}
$$

for the Lie expressions $s_{i}(X, Y)$ defined by

$$
\frac{d}{d t}[X, t X+Y, \ldots, t X+Y]=\sum s_{i}(X, Y) t^{i} \text { with } p-1 \text { factors ' } t X+Y^{\prime} .
$$

For example, if $p=2$ then $s_{1}(X, Y)=[X, Y]$, and if $p=3$ then $s_{1}(X, Y)=[Y, X, Y]$ and $s_{2}(X, Y)=[X, Y, X]$.

We adopt the convention that, in characteristic 0 , every Lie algebra is restricted with trivial $p$-mapping. This way, from now on we can uniformly work with restricted Lie algebras.

Recall that every restricted Lie algebra $L$ has a restricted universal enveloping algebra, a unital associative algebra $U_{p}(L)$ equipped with a map of restricted Lie algebras $L \rightarrow U_{p}(L)$, universal with respect to this property. The Lie bracket in $L$ is identified with the commutator $[x, y]=x y-y x$, and the $p$-mapping in $L$ is identified with the $p$-power operation in $U_{p}(L)$. The map $L \rightarrow U_{p}(L)$ is injective.

Recall next that a Hopf algebra is an associative algebra $R$ equipped with additional structure, in particular an augmentation $\varepsilon: R \rightarrow \mathbf{k}$ and a coproduct $\Delta: R \rightarrow R \otimes R$ which are algebra homomorphisms, and an antipode $S: R \rightarrow R$ which is an algebra antihomomorphism, subject to some axioms that we shall not need; see [40.

We will use the following classical facts, see [36. Theorem III.5.4 and Exercise 2].

Proposition 2.1. The (restricted) universal enveloping algebra $U(L)$, respectively $U_{p}(L)$, is a Hopf algebra. The augmentation, coproduct and antipode are given by

$$
\varepsilon: U(L) \rightarrow U(L) /\langle L\rangle \cong \mathbf{k} ; \quad \Delta(x)=x \otimes 1+1 \otimes x ; \quad S(x)=-x \quad \forall x \in L .
$$

In a Hopf algebra $H$, call $x \in H$ primitive if $\Delta(x)=x \otimes 1+1 \otimes x$; the primitive elements of $H$ form a Lie subalgebra $P$ of $H$. If the ring $\mathbf{k}$ is a $\mathbb{Z}$-free module, then the primitive elements in $U(L)$ coincide with $L$, while if $L$ is restricted and $\mathbf{k}$ is p-torsion then the primitive elements in $U_{p}(L)$ coincide with $L$; 
If a (restricted) Lie algebra over $\mathbf{k}$ is given by a (restricted) Lie algebra presentation, then by the universal property the same presentation, now as a presentation of algebras over k, defines its (restricted) universal enveloping algebra. In particular, $R_{\Gamma}$ is the (restricted) enveloping algebra of $L_{\Gamma}$ or $L_{\Gamma, p}$, respectively.

Proof of Theorem 1.4i. As a universal enveloping algebra, $R_{\Gamma}=U\left(L_{\Gamma}\right)$ is by Proposition 2.1 a Hopf algebra (this also appears in [35]), and its Lie subalgebra of primitive elements $P$ is equal to $L_{\Gamma}$ or $L_{\Gamma, p}$, when considered as subset of $R_{\Gamma}$ in the obvious way.

We note for later use the following standard constructions, see also [32].

Proposition 2.2. If $G$ is a group then the group ring $\mathbf{k} G$ is a Hopf algebra with augmentation, coproduct and antipode given as follows:

$\varepsilon: \mathbf{k} G \rightarrow \mathbf{k}$ induced by the map $G \rightarrow 1 ; \quad \Delta(g)=g \otimes g ; \quad S(g)=g^{-1} \forall g \in G$.

Furthermore, if $H$ is a Hopf algebra and $\varpi$ denotes its augmentation ideal $\operatorname{ker}(\varepsilon)$, then $\bigoplus_{n \geq 0} \varpi^{n} / \varpi^{n+1}$ is naturally a graded Hopf algebra.

\section{The Magnus map}

3.1. Filtrations and gradings. We first recall that, since the relations of $R_{\Gamma}$ and $S_{\Gamma}$ are homogeneous, these rings are naturally graded by setting $\operatorname{deg}(v)=1$ for all $v \in V$. We view $R_{\Gamma}$ as a ring of polynomials in partially commuting variables $v \in V$.

Let us consider the augmentation ideal $\varpi=\langle V\rangle$ in $R_{\Gamma}$. It consists of all polynomials without constant term. Note that $\varpi^{n}$ then consists of all polynomials with no terms of degree $<n$. We define a topology on $R_{\Gamma}$ by declaring the sets $\varpi^{n}$ to form a basis of neighbourhoods of 0 , and let $\overline{R_{\Gamma}}$ be the completion of $R_{\Gamma}$ in this topology. We thus have

$$
R_{\Gamma} \cong \bigoplus_{n \geq 0} \varpi^{n} / \varpi^{n+1}, \quad \overline{R_{\Gamma}} \cong \prod_{n \geq 0} \varpi^{n} / \varpi^{n+1} .
$$

We write $\bar{\varpi}$ for the closure of $\varpi$ in $\overline{R_{\Gamma}}$. It consists of all power series with vanishing constant term, and similarly $\bar{\varpi}^{n}$ consists of the power series with no terms of degree $<n$.

For comparison, consider the group ring $\mathbf{k} A_{\Gamma}$, and let $\varpi\left(A_{\Gamma}\right)$ denote the augmentation ideal of $\mathbf{k} A_{\Gamma}$; it is the ideal

$$
\left\langle g-1 \mid g \in A_{\Gamma}\right\rangle=\langle v-1 \mid v \in V\rangle .
$$

We topologize $\mathbf{k} A_{\Gamma}$ by declaring the $\varpi\left(A_{\Gamma}\right)^{n}$ to form a basis of neighbourhoods of the identity, and let $\overline{\mathbf{k} A_{\Gamma}}$ denote the corresponding completion. Moreover, let $\operatorname{gr}\left(\mathbf{k} A_{\Gamma}\right):=\bigoplus_{n \geq 0} \varpi\left(A_{\Gamma}\right)^{n} / \varpi\left(A_{\Gamma}\right)^{n+1}$ be the associated graded algebra. We isolate the main ingredient of Theorem 1.1.

Lemma 3.1. We have $R_{\Gamma} \cong \operatorname{gr}\left(\mathbf{k} A_{\Gamma}\right)$ as graded algebras via the natural map

$$
\alpha: R_{\Gamma} \rightarrow \operatorname{gr}\left(\mathbf{k} A_{\Gamma}\right) ; v_{j} \mapsto\left[\left(v_{j}-1\right)\right] \text { for } v_{j} \in V .
$$

Proof. The isomorphism between the degree- $n$ subspace of $R_{\Gamma}$ and $\varpi\left(A_{\Gamma}\right)^{n} / \varpi\left(A_{\Gamma}\right)^{n+1}$ can be proven by elementary considerations, since $\varpi\left(A_{\Gamma}\right)^{n} / \varpi\left(A_{\Gamma}\right)^{n+1}$ is generated by expressions $\left(v_{1}-1\right) \cdots\left(v_{n}-1\right)$.

However, here is a somewhat more elegant shortcut: as we noted in Propositions 2.1 and $2.2, \mathbf{k} A_{\Gamma}, \operatorname{gr}\left(\mathbf{k} A_{\Gamma}\right)$, and $R_{\Gamma}$ are all cocommutative Hopf algebras, with coproduct induced respectively by $\Delta(g)=g \otimes g$, by $\Delta([g-1])=[(g-1) \otimes 1+1 \otimes$ $(g-1)]$ for $g \in A_{\Gamma}$ and by $\Delta(v)=v \otimes 1+1 \otimes v$ for $v \in V$.

The map $\alpha: R_{\Gamma} \rightarrow \operatorname{gr}\left(\mathbf{k} A_{\Gamma}\right)$ is a well defined map of unital graded algebras because the defining commutation property for the $v_{j}$ in $R_{\Gamma}$ is satisfied for their images, and all these elements are of degree 1 . Moreover, we see that this map is a map of Hopf algebras. 
Finally, $\alpha$ is an isomorphism when restricted to the degree 1 subspaces, since

$$
\varpi / \varpi^{2} \cong \mathbf{k} V \cong\left(A_{\Gamma} /\left[A_{\Gamma}, A_{\Gamma}\right]\right) \otimes \mathbf{k} \cong \varpi\left(A_{\Gamma}\right) / \varpi\left(A_{\Gamma}\right)^{2}
$$

Here, the last isomorphism is the standard isomorphism of the first group homology $H^{1}\left(A_{\Gamma} ; \mathbf{k}\right)=A_{\Gamma} /\left[A_{\Gamma}, A_{\Gamma}\right] \otimes \mathbf{k}$ as $\varpi\left(A_{\Gamma}\right) / \varpi\left(A_{\Gamma}\right)^{2}$. We conclude by [27, Theorems 5.18 and 6.11 ] that $\alpha$ is an isomorphism: it is a map between cocommutative Hopf algebras both generated as algebras in degree 1 and the map is an isomorphism in degree 1. This shortcut already appears in 32 .

Remark 3.2. An alternative proof of Lemma 3.1 was kindly suggested to us by Jacques Darné: there are natural maps

$$
A_{\Gamma} \supset V \rightarrow \overline{R_{\Gamma}} \quad \text { and } \quad R_{\Gamma} \supset V \rightarrow \overline{\mathbf{k} A_{\Gamma}}
$$

which induce isomorphisms $\overline{\mathrm{k} A_{\Gamma}} \leftrightarrow \overline{R_{\Gamma}}$ by universal properties. Since gr $R_{\Gamma} \cong R_{\Gamma}$, the result (and the last statement of Proposition 3.7) follow.

Proof of Theorem 1.1. Lemma 3.1 gives an isomorphism between $\varpi\left(A_{\Gamma}\right)^{n} / \varpi\left(A_{\Gamma}\right)^{n+1}$ and the degree- $n$ part of $R_{\Gamma}$. Since $R_{\Gamma}$ is graded and not only filtered, its degree- $n$ part is $\varpi^{n} / \varpi^{n+1}$, so we get the desired isomorphism $\varpi^{n} / \varpi^{n+1} \cong \varpi\left(A_{\Gamma}\right)^{n} / \varpi\left(A_{\Gamma}\right)^{n+1}$ for each $n \in \mathbb{N}$.

3.2. The Magnus map. We turn to the fundamental tool we use in relating the group $A_{\Gamma}$ with the algebra $R_{\Gamma}$ : it is the "Magnus map"

$$
\mu:\left\{\begin{aligned}
A_{\Gamma} & \rightarrow 1+\bar{\varpi} \subseteq{\overline{R_{\Gamma}}}^{\times} \subseteq \overline{R_{\Gamma}} \\
v & \mapsto 1+v \text { for } v \in V
\end{aligned}\right.
$$

Here, ${\overline{R_{\Gamma}}}^{\times}$is the group of multiplicative units of $\overline{R_{\Gamma}}$. We have to map to the completion because we have to map $v^{-1}$ to $\mu(v)^{-1}=1-v+v^{2}-v^{3}+\cdots$ which is an infinite sum. It is immediate that the commutation relations between the $v \in V$ defining $A_{\Gamma}$ also hold between the $\mu(v)$, therefore $\mu$ is well defined.

It is easy to describe quite explicitly a basis of the polynomial ring in partially commuting variables $R_{\Gamma}$. This comes hand-in-hand with a kind of normal form for elements of $A_{\Gamma}$ :

Definition 3.3. A word $v_{1}^{e_{1}} \cdots v_{n}^{e_{n}}$ with $v_{i} \in V$ and $e^{i} \in \mathbb{Z}$ is called $\Gamma$-reduced if the number $n$ of factors $v_{i}^{e_{i}}$ cannot be reduced by application of any sequence of moves which are either

(M1) remove $v_{i}^{0}$,

(M2) replace the piece $v_{i}^{e_{i}} v_{i+1}^{e_{i+1}}$ by $v_{i}^{e_{i}+e_{i+1}}$ (if $v_{i}=v_{i+1}$ ), or

(M3) replace $v_{i}^{e_{i}} v_{i+1}^{e_{i+1}}$ by $v_{i+1}^{e_{i+1}} v_{i}^{e_{i}}$ (if $\left\{v_{i}, v_{i+1}\right\} \in E$ ).

Note that none of these moves increases the number of factors.

We then immediately get the

Lemma 3.4. The set of (M3)-equivalence classes of $\Gamma$-reduced words is a basis of $R_{\Gamma}$; more precisely, any set of representatives of (M3)-equivalence classes of reduced words of length $n$ forms a basis of the degree-n component of $R_{\Gamma}$.

In case $\mathbf{k}=\mathbb{Z}$, or more generally if $\mathbf{k}$ has characteristic 0 , it is known that the Magnus map $\mu$ is injective, see [41, Corollary 4.8]. We adapt this argument to $\mathbf{k}$ of non-zero characteristic, arriving at some of the original results of this note:

Lemma 3.5. Let $\mathbf{k}$ be a ring of characteristic $p>0$.

Consider $g \in A_{\Gamma}$. There exists a maximal $k \in \mathbb{N}$, and minimal $s_{1}, \ldots, s_{k} \in \mathbb{N}$, such that there is a $\Gamma$-reduced monomial $m=w_{1}^{p^{s_{1}}} \cdots w_{k}^{p^{s_{k}}}$ with non-zero coefficient in $\mu(g)$. This monomial is unique. Furthermore, if $v_{1}^{e_{1}} \cdots v_{n}^{e_{n}}$ is a reduced representative of $g$ then $n=k$ and $v_{1} \cdots v_{n}=w_{1} \cdots w_{k}$ and $p^{s_{i}} \mid e_{i}$ and the coefficient of $m$ in $\mu(g)$ is $\left(e_{1} p^{-s_{1}}\right) \cdots\left(e_{n} p^{-s_{n}}\right)$. 
Proof. Consider a $\Gamma$-reduced representative $v_{1}^{e_{1}} \cdots v_{n}^{e_{n}}$ of $g$. By definition,

$$
\mu\left(v_{1}^{e_{1}} \cdots v_{n}^{e_{n}}\right)=\left(1+v_{1}\right)^{e_{1}} \cdots\left(1+v_{n}\right)^{e_{n}}
$$

which is a possibly infinite (if one of the $e_{i}$ is less than 0 ) $\mathbb{F}_{p}$-linear combination of words over $V$. Write $e_{j}=p^{s_{j}} \ell_{j}$ so that $p$ does not divide $\ell_{j}$. Because we are in characteristic $p$, we have $\left(1+v_{j}\right)^{e_{j}}=\left(1+v_{j}^{p^{s_{j}}}\right)^{\ell_{j}}$.

We may now apply a variant of Magnus's original argument [23, Satz I]: multiplying out (using the power series for the inverse), we obtain a multiple of $v_{1}^{p^{s_{1}}} \cdots v_{n}^{p^{s_{n}}}$ precisely once, with coefficient $\ell_{1} \cdots \ell_{n} \neq 0 \in \mathbb{F}_{p}$. Other terms either have fewer syllables or larger exponents. The monomial $v_{1} \cdots v_{n}$ and all other monomials with the same number of syllables and possibly larger exponents are $\Gamma$-reduced, because any sequence of moves which would reduce one of them could be applied in the same way to the original $v_{1}^{e_{1}} \cdots v_{n}^{e_{n}}$ and would reduce its number of factors, as well. Therefore the term $v_{1}^{p^{s_{1}}} \cdots v_{n}^{p^{s_{n}}}$ indeed is uniquely determined as the $\Gamma$-reduced monomial in $\mu(g)$ with non-zero coefficient with maximal number of syllables and minimal exponents.

Since $\mu(g)$ is independent of the choice of representative of $g$, every other $\Gamma$ reduced representative $\left(v_{1}^{\prime}\right)_{1}^{e_{1}^{\prime}} \cdots\left(v_{n^{\prime}}^{\prime}\right) e_{n^{\prime}}^{\prime}$ must satisfy $n=n^{\prime}$ and $v_{1} \cdots v_{n}=v_{1}^{\prime} \cdots v_{n^{\prime}}^{\prime}$.

From this (and we note it for further use) we may deduce that every element of $A_{\Gamma}$ has an essentially unique reduced representative:

Proposition 3.6 ([41, Theorem 4.14]). If $v_{1}^{e_{1}} \cdots v_{n}^{e^{n}}$ and $w_{1}^{f_{1}} \cdots w_{m}^{f_{m}}$ are two reduced words representing the same element of $A_{\Gamma}$, then one can be obtained from the other by a finite number of applications of (M3). In particular, $n=m$.

Proof. We note first by Lemma 3.5 that $m=n$. We then proceed by induction on $m$. Consider the equal elements $v_{2}^{e_{2}} \cdots v_{m}^{e_{m}}$ and $v_{1}^{-e_{1}} w_{1}^{f_{1}} \cdots w_{m}^{f_{m}}$. The latter is not $\Gamma$-reduced, again by Lemma 3.5. so there must exist $k \in \mathbb{N}$ with $w_{k}=v_{1}$ and $\left\{v_{1}, w_{i}\right\} \in E$ for all $i \leq k$. If $f_{k} \neq e_{1}$ then $w_{1}^{f_{1}} \cdots w_{k}^{f_{k}-e_{1}} \cdots w_{m}^{f_{m}}$ is $\Gamma$-reduced, yet again contradicting Lemma 3.5, so $f_{k}=e_{1}$ and we apply induction to $v_{2}^{e_{2}} \cdots v_{m}^{e_{m}}$ and $w_{1}^{f_{1}} \cdots \widehat{w_{k}^{f_{k}}} \cdots w_{m}^{f_{m}}$, where the factor with hat is left out.

Proposition 3.7. For arbitrary $\mathbf{k}$, the Magnus map $\mu: A_{\Gamma} \rightarrow \overline{R_{\Gamma}}$ is injective.

It maps $\gamma_{n}\left(A_{\Gamma}\right)$ into the subgroup $1+\bar{\varpi}^{n}$ of $1+\bar{\varpi} \subset \overline{R_{\Gamma}}$. We get an induced map of graded Lie algebras

$$
\mu_{L}: \bigoplus_{n \geq 1} \gamma_{n}\left(A_{\Gamma}\right) / \gamma_{n+1}\left(A_{\Gamma}\right) \rightarrow \bigoplus_{n \geq 1}\left(1+\bar{\varpi}^{n}\right) /\left(1+\bar{\varpi}^{n+1}\right) \cong \bigoplus_{n \geq 1} \varpi^{n} / \varpi^{n+1} \subset R_{\Gamma},
$$

where the Lie algebra structure of $R_{\Gamma}$ is the one induced from the algebra structure.

The algebra map induced by $\mu$ on the group algebra $\mathbf{k} A_{\Gamma}$ extends continuously to an isomorphism of filtered associative $\mathbf{k}$-algebras

$$
\bar{\mu}: \overline{\mathbf{k} A_{\Gamma}} \cong \overline{R_{\Gamma}} \text {. }
$$

In particular,

$$
\mathbf{k} A_{\Gamma} / \varpi^{n}\left(A_{\Gamma}\right) \cong \overline{\mathbf{k} A_{\Gamma}} / \overline{\varpi^{n}\left(A_{\Gamma}\right)} \cong \overline{R_{\Gamma}} / \overline{\varpi^{n}}=R_{\Gamma} / \varpi^{n} \cong \operatorname{gr}\left(\mathbf{k} A_{\Gamma}\right) / \operatorname{gr}\left(\mathbf{k} A_{\Gamma}\right)_{\geq n},
$$

using Lemma 3.1 for the last isomorphism. As $\mathbf{k}$-modules, these are of course also isomorphic to $\left(R_{\Gamma}\right)_{<n} \cong \operatorname{gr}\left(\mathbf{k} A_{\Gamma}\right)_{<n}$.

Proof. Let $\mathbf{k}^{\prime}$ be the image of $\mathbb{Z}$ in $\mathbf{k}$; it is either $\mathbb{Z}$ or $\mathbb{Z} / N$ for some integer $N$. The case $\mathbb{Z}$ is already covered; if $\mathbf{k}^{\prime}=\mathbb{Z} / N$, let $p$ be a prime number dividing $N$. We prove the stronger statement that the composition $A_{\Gamma} \stackrel{\mu}{\rightarrow} \overline{R_{\Gamma}} \rightarrow \overline{R_{\Gamma} \otimes_{\mathbb{Z}} \mathbb{F}_{p}}$ is injective, i.e., we assume without loss of generality that $\mathbf{k}=\mathbb{F}_{p}$. Injectivity of $\mu$ for $\mathbf{k}=\mathbb{F}_{p}$ directly follows from Lemma 3.5. 
It is an elementary calculation in non-commutative power series that the $1+\bar{\varpi}^{n}$ form a central series of subgroups of $1+\bar{\varpi}$. By the minimality and functoriality of the lower central series,

$$
\gamma_{n}(1+\bar{\varpi}) \subseteq 1+\bar{\varpi}^{n} \quad \text { and then } \quad \mu\left(\gamma_{n}\left(A_{\Gamma}\right)\right) \subseteq 1+\bar{\varpi}^{n} .
$$

Elementary calculations in the non-commutative power series ring also show that we have an isomorphism of associated graded Lie algebras

$$
\bigoplus_{n \geq 1}\left(1+\bar{\varpi}^{n}\right) /(1+\varpi)^{n+1} \cong \bigoplus_{n \geq 1} \varpi^{n} / \varpi^{n+1} ;[1+w] \mapsto[w]
$$

where the right hand side is the graded Lie algebra structure underlying the associated graded algebra $R_{\Gamma}$ (with only the central summand $\varpi / \varpi^{1}$ of $R_{\Gamma}$ missing). As $R_{\Gamma}$ is already a graded algebra, it coincides with its associated graded. For details of these computations, compare e.g. [41, Lemma 4.10].

Finally, the induced algebra map $\mathrm{k} A_{\Gamma} \rightarrow \overline{R_{\Gamma}}$ is compatible with the augmentation homomorphisms as the same is true for the initial map $\mu: A_{\Gamma} \rightarrow 1+\bar{\varpi}$ (all elements on the left and on the right have augmentation 1). Consequently, it preserves the filtrations by powers of the augmentation ideals and induces a homomorphism $\operatorname{gr}(\mu)$ on the associated graded algebra. On the generating set $V$ this homomorphism is evidently the inverse of the map $\alpha$ of Lemma 3.1 .

We learn that our homomorphism of complete filtered algebras $\bar{\mu}: \overline{\mathbf{k} A_{\Gamma}} \rightarrow \overline{R_{\Gamma}}$ induces an isomorphism of the associated graded algebras. By general theory therefore $\bar{\mu}$ itself is an isomorphism. In more detail, $\overline{\mathrm{k} A_{\Gamma}}$ is the inverse limit of the $\overline{\mathbf{k} A_{\Gamma}} / \overline{\varpi^{n}\left(A_{\Gamma}\right)}$, and correspondingly for $\overline{R_{\Gamma}}$. Inductively and using the 5-lemma, $\bar{\mu} / \overline{\varpi^{n}}: \overline{\mathbf{k} A_{\Gamma}} / \overline{\varpi^{n}\left(A_{\Gamma}\right)} \rightarrow \overline{R_{\Gamma}} / \overline{\varpi^{n}}$ is an isomorphism (as $\bar{\mu} / \overline{\varpi^{n}}$ is the extension of $\bar{\mu} / \overline{\varpi^{n-1}}$ by the isomorphism $\left.\operatorname{gr}(\mu)_{n}\right)$. Finally, $\bar{\mu}$ is an isomorphism as limit of isomorphisms.

\section{Cohomology}

A (topological) way to define and compute the cohomology of a discrete group $G$ is via a classifying space $X_{G}$. By definition, this is a connected CW-cell complex with $\pi_{1}\left(X_{G}\right)=G$ whose universal covering is contractible. We then have $H^{*}(G ; \mathbf{k})=H^{*}\left(X_{G} ; \mathbf{k}\right)$.

Proof of Theorem 1.2, first claims. To compute the structure of the cohomology ring $H^{*}\left(A_{\Gamma} ; \mathbf{k}\right)$, we first show that $X_{\Gamma}$ of (1) is a (particularly nice) classifying space for $A_{\Gamma}$. The space $X_{\Gamma}$ inherits a CW-cell structure (indeed a cube complex structure) from the product cell structure of $\left(S^{1}\right)^{V}$, where $S^{1}$ has just one 0 -cell $\{*\}$ consisting of the base point and one 1-cell. Then $X_{\Gamma}$ has a single vertex $*^{V}$ and precisely one loop $\left(S^{1}\right)^{\{v\}} \times\{*\}^{V \backslash\{v\}}$ for each generator $v \in V$. The 2cells in $X_{\Gamma}$ give the commutation relations. By the standard computation of the fundamental group of CW-complexes (based on the van Kampen theorem) we then have $\pi_{1}\left(X_{\Gamma}, *^{V}\right)=A_{\Gamma}$.

Furthermore, the link of the single vertex in $X_{\Gamma}$ is a flag complex, since every subset of a clique is a clique. Therefore, $X_{\Gamma}$ is a cube complex whose link is a flag complex, so $X_{\Gamma}$ is a locally $\operatorname{CAT}(0)$ space [12], see [5, Theorem 5.18], so its universal cover is contractible.

The cells given in the expression of $X_{\Gamma}$ above form a basis of the homology of $X_{\Gamma}$ : the differentials in the cellular chain complex vanish identically, because every cell sits in a subcomplex which is the cellular chain complex of a torus with precisely this property. Note that we get a basis of $H_{*}\left(X_{\Gamma} ; \mathbf{k}\right)$ as free $\mathbf{k}$-module by the images of the fundamental classes of all subtori $T^{C}$ where $C$ runs through the cliques in $\Gamma$. As the homology is finitely generated free, the cohomology is canonically the dual of the homology. We see that $H^{*}\left(X_{\Gamma} ; \mathbf{k}\right)$ is precisely the quotient of the exterior algebra $H^{*}\left(T^{V} ; \mathbf{k}\right)=\bigwedge^{*}(\mathbf{k} V)$, the cohomology of the ambient torus $T^{V}$, by the submodule generated by all products $v_{1} \ldots v_{r}$ such that $v_{1}, \ldots, v_{r}$ do not span a 
clique in $\Gamma$. The comparison map is induced by the inclusion $X_{\Gamma} \hookrightarrow T^{V}$. That this map is surjective with the claimed kernel follows by naturality and the know (co)homology of $T^{V}$, together with the information about the rank of $H^{*}\left(X_{\Gamma} ; \mathbf{k}\right)$ we obtained from the cellular complex. Now the quotient algebra is precisely the algebra $S_{\Gamma}$ and we have proven $H^{*}\left(A_{\Gamma} ; \mathbf{k}\right)=H^{*}\left(X_{\Gamma} ; \mathbf{k}\right)=S_{\Gamma}$ as algebras.

We note that $H^{*}\left(X_{\Gamma} ; \mathbf{k}\right)=S_{\Gamma}$ has a natural $\mathbf{k}$-basis indexed by cliques $C$ in $\Gamma$ : a degree- $k$ basis element corresponding to a clique $C=\left\{v_{0}, \ldots, v_{k-1}\right\}$ is given by the product $v_{C}:=v_{k-1} \cdots v_{0}$ - to make this definite, we pick a total ordering of the vertices and write the factors in decreasing order.

4.1. Koszul algebras. Back to general theory, consider a graded associative algebra $R$ presented as $T(W) / I$ for a finitely generated free $\mathbf{k}$-module $W$, its tensor algebra $T(W)$ and an ideal $I \leq T(W)$. In case $I$ is generated by a subspace $I_{2}$ of $W^{\otimes 2}$, the algebra is called quadratic; and it then admits a quadratic dual $R^{!}:=T\left(W^{*}\right) /\left(I_{2}^{\perp}\right)$; here by $I_{2}^{\perp}$ we mean the subset of $\left(W^{*}\right)^{\otimes 2} \cong\left(W^{\otimes 2}\right)^{*}$ annihilating $I_{2}$. Clearly $R^{! !} \cong R$. Now, with $\mathbf{k} V$ the free $\mathbf{k}$-module with basis $V$, setting

$$
\begin{aligned}
& G_{R}:=\langle v \otimes w-w \otimes v \text { for }\{v, w\} \in E\rangle \subset \mathbf{k} V^{\otimes 2}, \\
& G_{S}:=\langle v \otimes w \text { for }\{v, w\} \notin E, v \otimes w+w \otimes v \text { for }\{v, w\} \in E\rangle \subset \mathbf{k} V^{\otimes 2},
\end{aligned}
$$

we have as algebras

$$
R_{\Gamma}=T(\mathbf{k} V) /\left\langle G_{R}\right\rangle \text { and } S_{\Gamma}=\bigwedge^{*}(\mathbf{k} V) /\langle v \wedge w \text { for }\{v, w\} \notin E\rangle=T(\mathbf{k} V) /\left\langle G_{S}\right\rangle .
$$

Let us identify $\mathbf{k} V^{\otimes 2}$ with $\left(\mathbf{k} V^{\otimes 2}\right)^{*}$ via the basis $\{v \otimes w \mid v, w \in V\}$ and its dual basis. Then $G_{S}$ is the annihilator of $G_{R}$ (they clearly annihilate each other, and the ranks add up to the total dimension $|V|^{2}$ ), and therefore $R_{\Gamma}$ and $S_{\Gamma}$ are quadratic duals of each other.

Returning to generality, recall that a quadratic algebra $R$ is called Koszul if its Koszul complex is acyclic, [24, 3.4.7]. We recall the Koszul complex (in our concrete situation) below and we mention that this is only one of a number of different equivalent characterizations of the Koszul property. It implies that the Yoneda algebra $\operatorname{Ext}_{R}(\mathbf{k}, \mathbf{k})$ is isomorphic to $R^{!}$, compare [31, Theorem 2.5].

Proof of Theorem 1.2, second claim. We now show that $R_{\Gamma}$ and $S_{\Gamma}$ are Koszul. Deliberately, we are a bit brief as we believe that this is mainly of interest to readers which have the required background. In fact, a quadratic algebra is Koszul if and only if its quadratic dual is [24, Proposition 3.4.8]. Therefore it suffices to prove the Koszul property for $S_{\Gamma}$, and there is a simple sufficient (but not necessary) condition, the existence of a quadratic Gröbner basis. Recall that a Gröbner basis for an ideal $I \leq \Lambda^{*}(\mathbf{k} V)$ is a set $G$ of generators for $I$ such that the leading terms (with respect to a compatible order of monomials) of elements of $G$ generate the same ideal as the leading terms of all elements of $I$. Now $G:=\{v \wedge w \mid\{v, w\} \notin$ $E\}$ is a Gröbner basis, as follows from Buchberger's criterion: "for all $f, f^{\prime} \in G$ whose respective leading terms $g, g^{\prime}$ have least common multiple $\ell$, the syzygy $(\ell / g) f-\left(\ell / g^{\prime}\right) f^{\prime}$ must vanish".

Alternatively and without using Gröbner basis, the work of Fröberg [11, in particular Section 3] also implies that $R_{\Gamma}$ (and $S_{\Gamma}$ ) are Koszul. His proof runs essentially as follows and uses directly the Koszul complex of $R_{\Gamma}$ which we now construct. Consider the right $R_{\Gamma}$-module $P_{*}=\operatorname{Hom}_{\mathbf{k}}\left(S_{\Gamma}, R_{\Gamma}\right)$. Recall that, qua k-module, $S_{\Gamma}$ is finitely generated free with basis indexed by cliques in $\Gamma$. Consequently, this basis induces and isomorphism $P_{*} \cong \bigoplus_{C} v_{C} R_{\Gamma}$, where the sum is over the cliques in $\Gamma$. It is bigraded by $S_{\Gamma^{-}}$and $R_{\Gamma^{-}}$degree. Consider the map $d: P_{*} \rightarrow P_{*}$ with

$$
d(f)(p)=\sum_{v \in V} v f(v p) \quad \text { for } f \in P_{*}, p \in S_{\Gamma} .
$$

In our basis, $d\left(\left(v_{k-1} \cdots v_{0}\right) \cdot r\right)=\sum(-1)^{j}\left(v_{k-1} \cdots \widehat{v_{j}} \cdots v_{0}\right) \cdot v_{j} r$. A direct computation shows that $d^{2}=0$. Note that $d$ increases the $R_{\Gamma}$-degree by 1 , and decreases 
the $S_{\Gamma}$-degree by 1 , so $\left(P_{*}, d\right)$ becomes a chain complex of finitely generated free

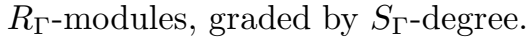

To prove acyclicity of the Koszul complex $\left(P_{*}, d\right)$ we define a chain contraction map $s: P_{*} \rightarrow P_{*+1}$ of $\mathbf{k}$-modules as follows. Recall that we have a $\mathbf{k}$-basis of $P_{*}$ given by elements $v_{C} \cdot w$ for a clique $C$ of $\Gamma$ and a basis element $w$ of $R_{\Gamma}$ given as a $\Gamma$-reduced monomial over $V$ according to Definition 3.3. To define $s\left(v_{C} \cdot w\right)$ we consider two cases. If we can write $w=v w^{\prime}$ in reduced form with $v \in V$ and with $w^{\prime}$ a word in letters from $V$ in such a manner that $v<\min C$ (for the total ordering on $V$ picked above) and such that $C \cup\{v\}$ is a clique of $\Gamma$, then we choose $v$ minimal with this property, and we set $s\left(v_{C} \cdot v w^{\prime}\right):=v_{C \cup\{v\}} \cdot w^{\prime}$. Otherwise, we set $s\left(v_{C} \cdot w\right):=0$.

We now carry out the elementary calculation to see that $s$ is a chain contraction, meaning $s d+d s=1-\epsilon$, where $\epsilon: P_{*} \rightarrow \mathbf{k}$ is the augmentation map, projecting onto the summand of bidegree $(0,0)$. For this, consider $x=v_{C} \cdot w$. The calculation splits into three cases.

(1) If $C=\emptyset$ and $w=1$, then $(s d+d s)(x)=0=(1-\epsilon)(x)$.

(2) Assume that $C=\left\{v_{0}, \ldots, v_{k}\right\} \neq \emptyset$ and $w$ cannot be written in the form $w=v w^{\prime}$ as above. Then

$$
d s(x)=0 \quad \text { while } \quad s d(x)=\sum(-1)^{j} s\left(\left(v_{k-1} \cdots \widehat{v}_{j} \cdots v_{0}\right) \cdot v_{j} w\right) .
$$

By hypothesis, no letter in $w$ can be swapped with $v_{j}$ and added to $C \backslash\left\{v_{j}\right\}$, so all summands vanish except the 0th which is $x$.

(3) Assume that $C=\left\{v_{0}, \ldots, v_{k}\right\}$ and $w$ can be written in the form $v_{-1} w^{\prime}$ such that $C \cup\left\{v_{-1}\right\}$ is a clique in $\Gamma$, with $v_{-1}<\min C$, chosen minimal among all such possibilities. Then $v_{-1}$ commutes with all $v_{j}$, so

$$
\begin{aligned}
& s d(x)=\sum(-1)^{j} s\left(v_{C \backslash\left\{v_{j}\right\}} \cdot v_{j} v_{-1} w^{\prime}\right)=\sum_{j=0}^{k-1}(-1)^{j} v_{C \backslash\left\{v_{j}\right\} \cup\left\{v_{-1}\right\}} \cdot v_{j} w^{\prime}, \\
& d s(x)=d\left(v_{C \cup\{v\}} \cdot w^{\prime}\right)=\sum_{j=-1}^{k-1}(-1)^{j+1} v_{C \backslash\left\{v_{j}\right\} \cup\left\{v_{-1}\right\}} \cdot v_{j} w^{\prime},
\end{aligned}
$$

and the terms cancel pairwise except the one with $j=-1$, giving again $(d s+s d)(x)=x$.

It follows that $P_{*}$ is a free $R_{\Gamma}$-resolution of $\mathbf{k}$.

We note that the usual definition of Koszul algebras is given over fields of characteristic 0; however, in our case, we need not impose any restriction on the commutative ring $\mathbf{k}$ (other than interpreting $(\mathbf{k} V)^{*}$ as naturally isomorphic to $\mathbf{k} V$ ), since the rings $R_{\Gamma}$ and $S_{\Gamma}$ are $\mathbf{k}$-free.

\section{Central series}

5.1. Labute's general theory. Labute gave in [21] a condition under which a presentation $\langle V \mid \mathcal{R}\rangle$ of a group $G$ determines a presentation of the associated Lie algebra $L(G):=\bigoplus_{n \geq 1} \gamma_{n}(G) / \gamma_{n+1}(G)$. Such a group presentation is now called "mild", and Anick gave in [1] a valuable criterion for this to happen: view all $r \in \mathcal{R}$ as elements of the free associative algebra $T(\mathbb{Z} V)$, under the Magnus embedding $F_{V} \rightarrow T(\mathbb{Z} V)$. Let $n$ be such that $r-1 \in \varpi^{n} \backslash \varpi^{n+1}$, and let $r^{\prime}$ denote the image of $r$ in the quotient $\varpi^{n} / \varpi^{n+1}$. Then $\langle V \mid \mathcal{R}\rangle$ is mild if and only if $\left\{r^{\prime}|r \in \mathcal{R}\rangle\right.$ is "inert". We need not define here the meaning of "inert" (a.k.a. "strongly free", see e.g. 16]), but merely note that there are powerful sufficient conditions guaranteeing that a set is inert in the free associative algebra, one of them being that it forms a Gröbner basis. It follows then quite generally that the Lie algebra $L(G)$ admits as presentation $\left\langle V \mid r^{\prime} \forall r \in \mathcal{R}\right\rangle$, see [21, Theorem 1]; and a similar statement holds for the restricted Lie algebra $\bigoplus_{n \geq 1} \lambda_{n, p}(G) / \lambda_{n+1, p}(G)$, 
see [21, Theorem 3]. Labute's conditions are non-trivial to check, so we shall in fact recover his results rather than use them.

5.2. First easy results for RAAGs. By Proposition 3.7 the rings $R_{\Gamma} / \varpi^{n}$ and $\mathbf{k} A_{\Gamma} / \varpi(G)^{n}$ are isomorphic, so the dimension subgroups $\delta_{n, \mu}$ and $\delta_{n, \mathbf{k} A_{\Gamma}}$ are equal. Furthermore, since the Magnus map $\mu$ has image in the subring of $R_{\Gamma}$ generated by 1 and $V$, the groups $\delta_{n, \mu}$ depend on $\mathbf{k}$ only via the image $\mathbf{k}^{\prime}$ of $\mathbb{Z}$ in $\mathbf{k}$.

We consider two cases: if $\mathbb{Z} \subseteq \mathbf{k}$ then the dimension subgroups associated with the rings $\mathbf{k}$ and $\mathbb{Q}$ agree. If, on the other hand, $\mathbb{F}_{p} \subseteq \mathbf{k}$, then the dimension subgroups associated with the rings $\mathbf{k}$ and $\mathbb{F}_{p}$ agree. In all cases, we reduce to the case $\mathbf{k} \in\left\{\mathbb{F}_{p}, \mathbb{Z}\right\}$.

Proof of Theorem 1.3i, We apply the classical results of Jennings and Hall. For $\mathbf{k}=\mathbb{Q}$ we have $\gamma_{n, 0}=\delta_{n, \mathbf{k} A_{\Gamma}}$; compare [15, 19] which treat the case of torsion-free nilpotent groups to which the general case easily reduces. For $\mathbf{k}=\mathbb{F}_{p}$ we have $\gamma_{n, p}=\delta_{n, \mathbf{k} A_{\Gamma}}$; compare [18] which treats the case of finite $p$-groups to which the general case easily reduces.

\section{Lie Algebras associated With $\Gamma$}

Recall that the cohomology of a Lie algebra $L$, defined as $\operatorname{Ext}_{U(L)}(\mathbf{k}, \mathbf{k})$, may be computed using its Chevalley complex $\left(\bigwedge^{*}\left(L^{\circ}\right), d\right)$, with $L^{\circ}$ the "small dual" of $L$, namely

$$
L^{\circ}=\left\{\phi \in L^{*} \mid \operatorname{ker} \phi \text { contains a finite-codimensional ideal }\right\},
$$

and the differential $d: L^{\circ} \rightarrow \bigwedge^{2} L^{\circ}$ is the dual of the Lie bracket map $\bigwedge^{2} L \rightarrow L$ (extended to all degrees by requiring $d$ to be a graded derivation). Note that $L^{\circ}$ is just so defined that the image of $d$ belongs to $\bigwedge^{2} L^{\circ} \subset\left(\bigwedge^{2} L\right)^{*}$. Since $\bigwedge^{*}\left(L^{\circ}\right)$ is a graded commutative algebra and $d$ is a derivation, the homology $\left(\bigwedge^{*}\left(L^{\circ}\right), d\right)$ is naturally a graded commutative algebra.

Proof of Theorem 1.4i, Lie algebra cohomology of $L_{\Gamma}$. The enveloping algebra of $L_{\Gamma}$ is $R_{\Gamma}$, which is Koszul with Koszul dual $S_{\Gamma}$, so we have

$$
H^{*}\left(L_{\Gamma} ; \mathbf{k}\right)=H^{*}\left(\bigwedge^{*}\left(L^{\circ}\right), d\right)=\operatorname{Ext}_{R_{\Gamma}}(\mathbf{k}, \mathbf{k})=S_{\Gamma} .
$$

Note that $\bigwedge^{*} L_{\Gamma}^{\circ}$ admits two gradings, one as an exterior algebra and one inherited from the grading of $L_{\Gamma}$. In $H^{*}\left(\bigwedge^{*}\left(L^{\circ}\right), d\right)$, these two gradings coincide - this is precisely the content of $S_{\Gamma}$ being a Koszul algebra.

In the following, we write $L$ for $L_{\Gamma}$ if the characteristic of $\mathbf{k}$ is 0 , and for $L_{\Gamma, p}$ if the characteristic of $\mathbf{k}$ is $p$, and view $L$ as a subset of $R_{\Gamma}=U(L)$. Following Magnus' method [25. Theorem 5.12], consider $x \in L_{n}$, i.e. homogeneous of degree $n$. Then $x$ is a linear combination (with coefficients in $\mathbf{k}$ ) of a collection of bracket arrangements $\phi_{i}=\phi_{i}\left(v_{1}, \ldots, v_{n}\right)$. The assignment

$$
L_{n} \ni \phi_{i} \mapsto \phi_{i}\left(v_{1}, \ldots, v_{n}\right) \in \gamma_{n} \subseteq A_{\Gamma}
$$

is well defined on the subset of bracket arrangements, since $[v, w]=1 \in A_{\Gamma}$ for each $\{v, w\} \in E$. It extends k-linearly to a map

$$
\nu: L_{n} \rightarrow \gamma_{n} / \gamma_{n+1} \otimes_{\mathbb{Z}} \mathbf{k}
$$

of $\mathbf{k}$-modules. This map is clearly surjective, since $\gamma_{n} / \gamma_{n+1}$ is spanned by $n$-fold bracket arrangements, for an arbitrary group. Furthermore, the composition $\mu_{L} \circ$ $\nu: L \rightarrow R_{\Gamma}$ with $\mu_{L}$ given in Proposition 3.7 is a Lie algebra map sending $v$ to $v$. Therefore this composition is the inclusion of $L$ into $R_{\Gamma}$ and is in particular injective. This implies that $\nu$ is an isomorphism with inverse the Magnus map $\mu_{L}$.

Proof of Theorem 1.3ii, characteristic 0 . Consider $\mathbf{k}=\mathbb{Z}$. Since $L_{\Gamma}$ is $\mathbb{Z}$-free, it follows in particular that $\gamma_{n}\left(A_{\Gamma}\right) / \gamma_{n+1}\left(A_{\Gamma}\right)$ is torsion-free for each $n$, and therefore $\gamma_{n, 0}\left(A_{\Gamma}\right)=\gamma_{n}\left(A_{\Gamma}\right)$ for all $n$. 
Proof of Theorem 1.4ii, first two claims.

The isomorphism $\nu$ identifies $L_{\Gamma}$ and $\bigoplus_{n \geq 1}\left(\gamma_{n} / \gamma_{n+1}\right) \otimes_{\mathbb{Z}} \mathbf{k}$.

Lemma 6.1. Consider $\mathbf{k}=\mathbb{Z}$, and define the ideal $\varpi_{p}=\langle p, V\rangle$ of $R_{\Gamma}$.

The associated graded ring $\bigoplus_{n \geq 0} \varpi_{p}^{n} / \varpi_{p}^{n+1}$ is isomorphic to $R_{\Gamma} \otimes_{\mathbb{Z}} \mathbb{F}_{p}[\pi]$, with $\pi$ of degree 1 mapped to $[p] \in \varpi_{p} / \varpi_{p}^{2}$ under the isomorphism.

Proof. Powers of $\varpi_{p}$ define a new filtration on $R_{\Gamma}$, in which $v \in V$ still has degree 1 , but in addition $p$ also has degree 1 ; thus for instance $p^{2} v^{3}$ belongs to the fifth term of the filtration. The ring $R_{\Gamma}$ is $\mathbb{Z}$-free. When passing to the associated graded ring for the new grading, we get on the one hand $\bigoplus \varpi_{p}^{n} / \varpi_{p}^{n+1}$. On the other hand, this graded ring is obtained from the old associated graded (which is the graded algebra $R_{\Gamma}$ ) by replacing each copy of $\mathbb{Z}$ by its own associated graded under the filtration $\left(p^{n}\right)$, namely by $\mathbb{F}_{p}[\pi]$. This replacement amounts to tensoring over $\mathbb{Z}$ with $\mathbb{F}_{p}[\pi]$.

In case $p \geq 3$, we are now ready to identify the non-restricted Lie algebra $\bigoplus_{n \geq 1} \lambda_{n, p} / \lambda_{n+1, p}$ with $L_{\Gamma} \otimes_{\mathbb{Z}} \mathbb{F}_{p}[\pi]$. Let us temporarily write $\beta_{n}:=\mu^{-1}\left(1+\bar{\varpi}_{p}^{n}\right)$. We make the following claim.

Lemma 6.2. For $p \geq 3$ prime, the Magnus map $\mu$ induces a composition of (nonrestricted) Lie algebra isomorphisms over $\mathbb{F}_{p}[\pi]$, still written $\mu_{L}$,

$$
\mu_{L}: \bigoplus_{n \geq 1} \lambda_{n, p} / \lambda_{n+1, p} \rightarrow \bigoplus_{n \geq 1} \beta_{n} / \beta_{n+1} \rightarrow L_{\Gamma} \otimes_{\mathbb{Z}} \mathbb{F}_{p}[\pi]
$$

with the first map induced by inclusion $\lambda_{n, p} \leq \beta_{n}$ and the second map induced by $\beta_{n} / \beta_{n+1} \ni[1+a] \mapsto a \in \varpi_{p}^{n} / \varpi_{p}^{n+1}$.

In particular, we have $\beta_{n}=\lambda_{n, p}$.

Proof. To check that the first map is well-defined, it suffices to show $\lambda_{n, p} \leq \beta_{n}$. We have $\varpi_{p}^{n}=\sum_{m+i>n} p^{i} \varpi^{m}$. Consider $g \in \gamma_{m}$, so by definition $\mu(g)=1+x$ for some $x \in \varpi^{m}$. We then have $\mu\left(g^{p^{i}}\right)=(1+x)^{p^{i}}=1+p^{i} x+\cdots \in 1+\varpi_{p}^{m+i}$, so $\mu\left(\gamma_{m}^{p^{i}}\right) \subseteq 1+\varpi_{p}^{m+i}$. Since $\lambda_{n, p}=\prod_{m+i \geq n} \gamma_{m}^{p^{i}}$, we have shown $\lambda_{n, p} \leq \beta_{n}$.

Because the Magnus map $\mu: A_{\Gamma} \rightarrow 1+\bar{\varpi} \subset R_{\Gamma}$ is injective by Proposition 3.7 . so is the induced map $\beta_{n} / \beta_{n+1} \rightarrow\left(1+\bar{\varpi}_{p}^{n}\right) /\left(1+\varpi_{p}^{n+1}\right)=\varpi_{p}^{n} / \varpi_{p}^{n+1}$, which is our second map.

Since $p \geq 3$, the assignment $\pi \cdot[g]:=\left[g^{p}\right]$ for $g \in \lambda_{n, p}$ (with $\left.g^{p} \in \lambda_{n+1, p}\right)$ gives $\bigoplus \lambda_{n, p} / \lambda_{n+1, p}$ the structure of an $\mathbb{F}_{p}[\pi]$-module. For this we use the HallPetrescu identities [14, Theorems 3.1, 3.2]: if $g, h$ belong to an arbitrary group $G$, then $(g h)^{p} \equiv g^{p} h^{p}[h, g]^{n} \alpha(g, h)$ with $n=\left(\begin{array}{c}p \\ 2\end{array}\right)$ and $\alpha(\cdot, \cdot)$ a universal expression in $\gamma_{3}$. This implies $(g h)^{p}=g^{p} h^{p} \bmod \gamma_{n+2, p}$ for $g, h \in \gamma_{n, p}$ if either $n \geq 2$ or $n=1$ and $p \geq 3$. However, beware that if $n=1$ and $p=2$ then this does not hold in general, so the $p$-power operation is not linear. We see that $\mu_{L}$ maps this $p$-power operation to multiplication by $\pi$ on $L_{\Gamma} \otimes_{\mathbb{Z}} \mathbb{F}_{p}[\pi]$. It follows that $\mu_{L}$ is an $\mathbb{F}_{p}[\pi]$-Lie algebra homomorphism. Its image contains $V$ which generates $L_{\Gamma}$, so $\mu_{L}$ is surjective. Finally, $L_{\Gamma} \otimes_{\mathbb{Z}} \mathbb{F}_{p}[\pi]$ is the free Lie algebra over $\mathbb{F}_{p}[\pi]$ modulo the relations $[v, w]=0$ for $\{v, w\} \in E$. Those relations are clearly satisfied in the $\mathbb{F}_{p}[\pi]$-Lie algebra $\bigoplus_{n>1} \lambda_{n, p} / \lambda_{n+1, p}$, so the map $\mu_{L}$ is an isomorphism.

It then follows that the second map is surjective and therefore an isomorphism, so the first is also bijective, from which we deduce $\beta_{n}=\lambda_{n, p}$.

Proof of Theorem 1.3ii, characteristic $p$. Let $\mathbf{k}$ be an algebra over $\mathbb{F}_{p}$. By $[32$, the Lie algebra $\bigoplus_{n>1}\left(\gamma_{n, p} / \gamma_{n+1, p}\right) \otimes_{\mathbb{Z}} \mathbf{k}$ is isomorphic to the primitive subalgebra of $\bigoplus_{n \geq 0} \varpi\left(A_{\Gamma}\right)^{n} / \varpi\left(A_{\Gamma}\right)^{n+1} \cong R_{\Gamma}$, namely to $L_{\Gamma, p}$.

Proof of Theorem 1.4ii, last claim. This is precisely Lemma 6.2. 


\section{Growth SERIES}

We derive now some relations between the Poincaré series of $S_{\Gamma}, R_{\Gamma}, L_{\Gamma}$ and $L_{\Gamma, p}$ from general considerations. We recall that, for a graded algebra $R=\bigoplus_{n \geq 0} R_{n}$, its Poincaré series is $\Phi_{R}(t)=\sum_{n \geq 0} \operatorname{rank}\left(R_{n}\right) t^{n}$.

Proof of Theorem 1.5. First, we use Koszul duality between $R_{\Gamma}$ and $S_{\Gamma}$ to deduce $\Phi_{R_{\Gamma}}(t) \cdot \Phi_{S_{\Gamma}}(-t)=1$, compare [24, Theorem 3.5.1]. This relationship between the Poincaré series of $R_{\Gamma}$ and $S_{\Gamma}$ was already noted in [6, 38.

We have $\Phi_{S_{\Gamma}}(t)=\sum_{n \geq 0} \operatorname{rank} H^{n}\left(A_{\Gamma}, \mathbf{k}\right) t^{n}=\sum_{n \geq 0} c_{n}(\Gamma) t^{n}$, with $c_{n}(\Gamma)$ the number of $n$-cliques in $\Gamma$, from our explicit basis of $S_{\Gamma}$ given in Section 4 .

The relation between $\Phi_{R_{\Gamma}}$ and $\Phi_{L_{\Gamma}}$ is given by the Poincaré-Birkhoff-Witt theorem, namely the fact that $R_{\Gamma}$ and the symmetric algebra over $L_{\Gamma}$, respectively the degree- $p$ truncated symmetric algebra over $L_{\Gamma, p}$, are isomorphic as graded kmodules. It is expressed by the relation

$$
\sum_{n \geq 0} a_{n} t^{n}=\prod_{n \geq 1}\left(\frac{1}{1-t^{n}}\right)^{b_{n}}=\prod_{n \geq 1}\left(\frac{1-t^{p n}}{1-t^{n}}\right)^{c_{n}}
$$

if $\Phi_{R_{\Gamma}}(t)=\sum_{n \geq 0} a_{n} t^{n}, \Phi_{L_{\Gamma}}(t)=\sum_{n \geq 1} b_{n} t^{n}$, and $\Phi_{L_{\Gamma, p}}(t)=\sum_{n \geq 1} c_{n} t^{n}$.

Finally, we consider the growth series of the group $A_{\Gamma}$. It is the function $\Phi_{A_{\Gamma}}(t)=$ $\sum_{g \in A_{\Gamma}} t^{\|g\|}$, with $\|g\|$ the minimal number of terms of $V \cup V^{-1}$ required to write $g$ as a product. We cite [2]:

$$
\Phi_{A_{\Gamma}}(t)=\Phi_{R_{\Gamma}}\left(\frac{2 t}{1+t}\right) .
$$

Indeed, as we saw in Proposition 3.6, every element $g \in A_{\Gamma}$ can be written in the form $g=v_{1}^{e_{1}} \cdots v_{n}^{e_{n}}$ for some $e_{i} \in \mathbb{Z} \backslash\{0\}$ as a word of minimal length; and this expression is unique up to permuting some terms according to rule (M3). Let $I$ be the set of (M3)-equivalence classes $\left(v_{1}, \ldots, v_{n}\right)$ of minimal-length sequences. For an element $\left[v_{1}, \ldots, v_{n}\right]$ of $I$, the collection of all such terms $v_{1}^{e_{1}} \cdots v_{n}^{e_{n}}$ contributes $\left(t+t^{2}+t^{3}+\cdots\right)^{n}=(t /(1-t))^{n}$ to the growth series of $R_{\Gamma}$ because each $e_{i}$ can be an arbitrary positive natural number; and it contributes $(2 t /(1-t))^{n}$ to the growth of $A_{\Gamma}$, taking into account the signs of the $e_{i}$. Since we obtain all elements of $A_{\Gamma}$ and all basis elements of $R_{\Gamma}$ that way, we have

$$
\Phi_{A_{\Gamma}}(t)=\sum_{I}\left(\frac{2 t}{1-t}\right)^{n}=\sum_{I}\left(\frac{2 t /(1+t)}{1-2 t /(1+t)}\right)^{n}=\Phi_{R_{\Gamma}}\left(\frac{2 t}{1+t}\right),
$$

using $2 t /(1-t)=(2 t /(1+t)) /(1-2 t /(1+t)) \mathrm{y}$. We have finished the proof of Theorem 1.5 .

\section{MalceV Completions}

In this section we fix $\mathbf{k}=\mathbb{Q}$. Recall from [29] that a Malcev Lie algebra is a Lie algebra $L$ over $\mathbb{Q}$, given with a descending filtration $\left(L_{n}\right)_{n \geq 1}$ of ideals such that $L$ is complete with respect to the associated topology, and satisfying $L_{1}=L$ and $\left[L_{m}, L_{n}\right] \subseteq L_{m+n}$ and such that $\bigoplus_{n \geq 1} L_{n} / L_{n+1}$ is generated in degree 1 . Every Malcev Lie algebra admits an associated exponential group $\exp (L)$, which is $L$ as a set, with product given by the Baker-Campbell-Hausdorff formula $x \cdot y=$ $x+y+[x, y] / 2+\cdots$.

Lazard proved in 22] that every group homomorphism $\rho: G \rightarrow \exp (L)$ induces a morphism of graded Lie algebras $\bigoplus_{n \geq 1} \gamma_{n} / \gamma_{n+1} \otimes \mathbb{Q} \rightarrow \bigoplus_{n \geq 1} L_{n} / L_{n+1}$.

A Malcev completion of a group $G$ is a homomorphism $\rho: G \rightarrow \exp (L)$ for a Malcev Lie algebra $L$, universal in the sense that every representation $G / \gamma_{n} \rightarrow$ $\exp \left(L^{\prime}\right)$ for a (nilpotent) Malcev Lie algebra $L^{\prime}$ factors uniquely through $\exp \left(L / L_{n}\right)$; see [29, Definition 2.3].

Quillen gave a direct construction of the Malcev completion of a group in [32,33]: let $\overline{\mathbb{Q} G}=\operatorname{proj} \lim \mathbb{Q} G / \varpi^{n}$ be the completion of the group ring; then $\overline{\mathbb{Q} G}$ is a 
complete Hopf algebra. Let $L$ be its Lie subalgebra of primitive elements; it is a Malcev Lie algebra for the filtration $L_{n}=L \cap \bar{\varpi}^{n}$. Let exp: $L \rightarrow \overline{\mathbb{Q} G}$ be the usual power series map $\exp (x)=1+x+x^{2} / 2+\cdots$ which makes sense in $\overline{\mathbb{Q} G}$. Then its image $\bar{G}:=\exp (L)$ is a subgroup of the group of multiplicative units. It identifies with the Lie group associated to the Malcev Lie algebra $L$, and it consists precisely of the grouplike elements in $\overline{\mathbb{Q} G}$, namely the $g \in 1+\bar{\varpi}$ satisfying $\Delta(g)=g \otimes g$. The representation $\rho: G \rightarrow \exp (L) ; g \mapsto g$ is the Malcev completion of $G$.

The Magnus map $\mu: A_{\Gamma} \rightarrow{\overline{R_{\Gamma}}}^{\times}$yields an isomorphism of associative algebras $\overline{\mathbb{Q} A_{\Gamma}} \cong \overline{R_{\Gamma}}$. Both algebras are actually complete Hopf algebras, but the Magnus isomorphism does not preserve the Hopf algebra structure: $v \in V \subset \overline{\mathbb{Q} A_{\Gamma}}$ is grouplike, meaning $\Delta(v)=v \otimes v$ while $v \in V \subset \overline{R_{\Gamma}}$ is primitive, meaning $\Delta(v)=v \otimes 1+$ $1 \otimes v ;$ so $\Delta(\mu(v))=\Delta(1+v)=1 \otimes 1+v \otimes 1+1 \otimes v$ while $(\mu \otimes \mu)(\Delta(v))=(1+v) \otimes(1+v)$.

The Magnus map $\mu$ is, in fact, the truncation to order 1 of a Hopf algebra isomorphism $\mu_{\text {exp }}: \overline{\mathbb{Q} A_{\Gamma}} \rightarrow \overline{R_{\Gamma}}$, given on $v \in V$ by the classical exponential series

$$
\mu_{\exp }(v)=\sum_{n \geq 0} \frac{v^{n}}{n !}=1+v+\mathcal{O}\left(v^{2}\right) .
$$

Proof of Theorem 1.6. The proof that $\mu_{\exp }$ is an isomorphism of filtered associative algebras is exactly the same as that of Theorem 1.3ii, and will not be repeated. On the other hand, the fact that $\mu_{\exp }$ is a coalgebra map follows formally from the fact that the power series exp maps primitive elements to group-like elements:

$$
\begin{aligned}
\Delta\left(\mu_{\exp }(v)\right) & =\Delta\left(\sum_{n \geq 0} v^{n} / n !\right)=\sum_{n \geq 0} \Delta(v)^{n} / n ! \\
& =\sum_{n \geq 0} \frac{(v \otimes 1+1 \otimes v)^{n}}{n !}=\sum_{\ell, m \geq 0} \frac{(v \otimes 1)^{\ell}(1 \otimes v)^{m}}{\ell ! m !} \\
& =(\exp v \otimes 1)(1 \otimes \exp v)=\left(\mu_{\exp } \otimes \mu_{\exp }\right)(\Delta(v)) .
\end{aligned}
$$

We have proven the first claim.

It now suffices to use this isomorphism $\mu_{\text {exp }}$ to make even more concrete the construction of Quillen sketched above: in $\overline{\mathbb{Q} A_{\Gamma}}$ the space of primitive elements is slightly mysterious, for example, it contains

$$
\log (g)=\log (1-(1-g))=-\sum_{n \geq 1}(1-g)^{n} / n \quad \forall g \in A_{\Gamma} .
$$

In contrast to this, its exponential is the Malcev completion naturally containing $A_{\Gamma}$. In $\overline{R_{\Gamma}}$ the opposite holds: the space of primitive elements is the Lie subalgebra $L_{\Gamma}$ while its exponential cannot be better defined than as the exponential of $L_{\Gamma}$.

In all cases, the Hopf algebra isomorphism $\mu_{\text {exp }}$ directly yields the remaining claims of Theorem 1.6 .

We now turn to formality in the sense of Sullivan in rational homotopy theory. A finite CW-complex $X$ is called formal if its algebraic minimal model is quasiisomorphic to $\left(H^{*}(X ; \mathbb{Q}), 0\right)$. This implies that the rational homotopy type of $X$ is determined in a precise way by its rational cohomology ring. For details on rational homotopy theory compare [39] or the more recent [10].

We finally prove that the space $X_{\Gamma}$ constructed in Section 4 is formal. Recall that we defined $X_{\Gamma}$ as a (cubical) subspace of the smooth manifold $(\mathbb{R} / \mathbb{Z})^{V}$. It makes perfect sense to restrict smooth differential forms on $(\mathbb{R} / \mathbb{Z})^{V}$ to $X_{\Gamma}$. We define $A^{*}\left(X_{\Gamma}\right)$ to be the algebra of all such restrictions; it is a commutative differential graded algebra (cdga). It is an easy exercise that this cdga is quasi-isomorphic to the standard cdga over $\mathbb{R}$ of rational homotopy theory associated to $X_{\Gamma}$. There are basic one-forms $d x_{v}$ on $(\mathbb{R} / \mathbb{Z})^{V}$ coming from the obvious coordinate functions, for $v \in V$. Their images in $A^{*}\left(X_{\Gamma}\right)$ generate a sub-cdga with trivial differential, whose homology is $H^{*}\left(X_{\Gamma} ; \mathbb{R}\right)$ by Theorem 1.2. The inclusion of this sub-cdga in $A^{*}\left(X_{\Gamma}\right)$ is a quasi-isomorphism, showing that $X_{\Gamma}$ is formal. 
We now explicitly exhibit a minimal model for $X_{\Gamma}$. Recall from Section 6 the Chevalley complex $\left(\bigwedge^{*}\left(L_{\Gamma}^{\circ}\right), d\right)$ of $L_{\Gamma}$. Note that $L_{\Gamma}$ is graded, and $L_{\Gamma}^{\circ}$ may be identified with the graded dual of $L_{\Gamma}$. Consequently, there is a natural map $L_{\Gamma}^{\circ} \rightarrow$ $\mathbb{Q} V$ given by restricting to the degree-1 part. This map induces a map of graded algebras $\bigwedge^{*}\left(L_{\Gamma}^{\circ}\right) \rightarrow \bigwedge^{*}(\mathbb{Q} V) /\langle v \wedge w$ for $\{v, w\} \notin E\rangle=S_{\Gamma}$. Even better, this is a map of cdgas from the Chevalley complex to $S_{\Gamma}$, the latter equipped with zero differential, and indeed is a quasi-isomorphism. These are manifestations of the Koszul duality of $S_{\Gamma}$ and $R_{\Gamma}=U\left(L_{\Gamma}\right)$. As $X_{\Gamma}$ is formal and $S_{\Gamma}=H^{*}\left(X_{\Gamma} ; \mathbb{Q}\right)$ we conclude that $\Lambda^{*}\left(L_{\Gamma}^{\circ}, d\right)$ is a minimal model of $X_{\Gamma}$.

Here is yet an alternative proof: a group is called 1-formal if its Malcev Lie algebra is quadratic. It therefore follows from Theorem [1.6 that $A_{\Gamma}$ is 1-formal. The cohomology ring $H^{*}\left(X_{\Gamma} ; \mathbb{Q}\right) \cong S_{\Gamma}$ is Koszul by Theorem 1.2, so $X_{\Gamma}$ is formal by [30, Proposition 2.1].

\section{OutLOOK}

9.1. Subgroup growth. Baik, Petri, and Raimbault determined the subgroup growth of $A_{\Gamma}$ in terms of the graph $\Gamma$. Define $s_{n}\left(A_{\Gamma}\right)$ as the number of subgroups of $A_{\Gamma}$ of index precisely $n$. Then [4, Theorem A] establishes

$$
\lim _{n \rightarrow \infty} \frac{\log \left(s_{n}\left(A_{\Gamma}\right)\right)}{n \log (n)}=\alpha(\Gamma)-1,
$$

i.e. $s_{n}\left(A_{\Gamma}\right)$ grows like $(n !)^{\alpha(\Gamma)-1}$. Here, $\alpha(\Gamma)$ is the independence number of $\Gamma$, the largest number of vertices such that the full subgraph of $\Gamma$ spanned by them is discrete.

We do not discuss the rather complicated proof here. We leave it an open question to find a corresponding result for the growth of the number of finite index Lie subalgebras of $L_{\Gamma}$. Indeed, we expect that these two series are closely related and that the latter is slightly easier to control than $\left(s_{n}\left(A_{\Gamma}\right)\right)_{n \in \mathbb{N}}$.

We have identified $\gamma_{n, p}\left(A_{\Gamma}\right)$ with $\delta_{n, \mathbb{F}_{p_{p}} A_{\Gamma}}$ in Theorem 1.3ii For a group $G$, we could define $\gamma_{n, p^{e}}$ as the subgroup generated by $\gamma_{n}$ and all $\gamma_{i}^{p^{j}}$ with $i p^{j} \geq n p^{e-1}$. When $G$ is free, it was shown by Lazard that $\gamma_{n, p^{e}}(G)$ coincides with the dimension subgroup $\delta_{n, \mathbb{Z} / p^{e} \mathbb{Z}[G]}$ while this does not hold for general $G$, see [28].

We leave it as an exercise to extend Lazard's result to $A_{\Gamma}$.

9.2. Homology gradients. Given a group $G$ and a nested sequence of finite index normal subgroups $G_{n} \triangleleft G$ with $\bigcup_{n} \Gamma_{n}=\{1\}$, one defines for a field k the k-homology gradients

$$
b_{i}^{(2)}(G ; \mathbf{k}):=\limsup _{n} \frac{b_{i}\left(G_{n} ; \mathbf{k}\right)}{\left[G: G_{n}\right]} \text { for } i \in \mathbb{N} .
$$

For general groups $G$, it is unclear whether this quantity depends on the particular chain $\left\{G_{n}\right\}$. Until recently, it was also unclear in which manner this quantity depends on the coefficients k. Avradmidi, Okun, and Schreve in [3] use the classifying space $X_{\Gamma}$ and induced cell structures for coverings to explicitly compute these homology gradients. Let $F_{\Gamma}$ be the flag complex generated by $\Gamma$, i.e. the largest simplicial complex with vertex set $V$ and edge set $E$. Then

$$
b_{i}^{(2)}\left(A_{\Gamma} ; \mathbf{k}\right)=\overline{b_{i-1}}\left(F_{\Gamma} ; \mathbf{k}\right)
$$

where $\overline{b_{*}}\left(F_{\Gamma} ; \mathbf{k}\right)$ denotes the dimension of the reduced homology of $F_{\Gamma}$. In particular, for RAAGs the homology gradient is independent of the chain of normal subgroups, even though in many examples it does depend on the field of coefficients $\mathbf{k}$.

\section{REFERENCES}

[1] David J. Anick, Inert sets and the Lie algebra associated to a group, J. Algebra 111 (1987), no. 1, 154-165, DOI 10.1016/0021-8693(87)90246-8. MR913201

[2] Jayadev S. Athreya and Amritanshu Prasad, Growth in Right-Angled Groups and Monoids (2014), available at arXiv:1409.4142 preprint. 
[3] Grigori Avramidi, Boris Okun, and Kevin Schreve, Mod p and torsion homology growth in nonpositive curvature, 2020. arXiv:2003.01020.

[4] Hyungryul Baik, Bram Petri, and Jean Raimbault, Subgroup growth of right-angled Artin and Coxeter groups, 2018. preprint.

[5] Martin R. Bridson and André Haefliger, Metric spaces of non-positive curvature, SpringerVerlag, Berlin, 1999. MR2000k:53038

[6] Pierre Cartier and Dominique Foata, Problèmes combinatoires de commutation et réarrangements, Lecture Notes in Mathematics, No. 85, Springer-Verlag, Berlin-New York, 1969 (French). MR0239978

[7] Carl Gordon Arthur Droms, GRAPH GROUPS (ALGEBRA, KIM, ROUSH, MAGNUS), ProQuest LLC, Ann Arbor, MI, 1983. Thesis (Ph.D.)-Syracuse University. MR2633165

[8] Gérard H. E. Duchamp and Daniel Krob, The lower central series of the free partially commutative group, Semigroup Forum 45 (1992), no. 3, 385-394, DOI 10.1007/BF03025778. MR1179860

[9] - The free partially commutative Lie algebra: bases and ranks, Adv. Math. 95 (1992), no. 1, 92-126, DOI 10.1016/0001-8708(92)90045-M. MR1176154

[10] Yves Félix, Stephen Halperin, and Jean-Claude Thomas, Rational homotopy theory, Graduate Texts in Mathematics, vol. 205, Springer-Verlag, New York, 2001. MR1802847

[11] Ralph Fröberg, Determination of a class of Poincaré series, Math. Scand. 37 (1975), no. 1, 29-39, DOI 10.7146/math.scand.a-11585. MR0404254

[12] Mikhail L. Gromov, Hyperbolic groups, Essays in group theory, Math. Sci. Res. Inst. Publ., vol. 8, Springer, New York, 1987, pp. 75-263, DOI 10.1007/978-1-4613-9586-7_3. MR919829

[13] Karl W. Gruenberg, Residual properties of infinite soluble groups, Proc. London Math. Soc. (3) 7 (1957), 29-62, DOI 10.1112/plms/s3-7.1.29. MR0087652

[14] Philip Hall, A Contribution to the Theory of Groups of Prime-Power Order, Proc. London Math. Soc. (2) 36 (1934), 29-95, DOI 10.1112/plms/s2-36.1.29. MR1575964

[15] The Edmonton notes on nilpotent groups, Queen Mary College Mathematics Notes, Mathematics Department, Queen Mary College, London, 1969. MR0283083

[16] Stephen Halperin and Jean-Michel Lemaire, Suites inertes dans les algèbres de Lie graduées ("Autopsie d'un meurtre. II"), Math. Scand. 61 (1987), no. 1, 39-67, DOI 10.7146/math.scand.a-12190 (French). MR929396

[17] Nathan Jacobson, Restricted Lie algebras of characteristic p, Trans. Amer. Math. Soc. 50 (1941), 15-25, DOI 10.2307/1989908. MR0005118

[18] Stephen A. Jennings, The structure of the group ring of a p-group over a modular field, Trans. Amer. Math. Soc. 50 (1941), 175-185.

[19] - The group ring of a class of infinite nilpotent groups, Canad. J. Math. 7 (1955), 169-187, DOI 10.4153/CJM-1955-022-5. MR0068540

[20] Michael Kapovich and John J. Millson, On representation varieties of Artin groups, projective arrangements and the fundamental groups of smooth complex algebraic varieties, Inst. Hautes Études Sci. Publ. Math. 88 (1998), 5-95 (1999). MR1733326

[21] John P. Labute, The determination of the Lie algebra associated to the lower central series of a group, Trans. Amer. Math. Soc. 288 (1985), no. 1, 51-57, DOI 10.2307/2000425. MR773046

[22] Michel Lazard, Sur les groupes nilpotents et les anneaux de Lie, Ann. École Norm. Sup. (3) 71 (1954), 101-190.

[23] Wilhelm Magnus, Beziehungen zwischen Gruppen und Idealen in einem speziellen Ring, Math. Ann. 111 (1935), no. 1, 259-280, DOI 10.1007/BF01472217 (German). MR1512992

[24] Jean-Louis Loday and Bruno Vallette, Algebraic operads, Grundlehren der Mathematischen Wissenschaften [Fundamental Principles of Mathematical Sciences], vol. 346, Springer, Heidelberg, 2012. MR2954392

[25] Wilhelm Magnus, Abraham Karrass, and Donald Solitar, Combinatorial group theory, Second revised edition, Dover Publications, Inc., New York, 1976. Presentations of groups in terms of generators and relations. MR0422434

[26] Anatoliǔ I. Mal'cev, Nilpotent torsion-free groups, Izvestiya Akad. Nauk. SSSR. Ser. Mat. 13 (1949), 201-212 (Russian). MR0028843

[27] John W. Milnor and John C. Moore, On the structure of Hopf algebras, Ann. of Math. (2) 81 (1965), 211-264. MR0174052 (30 \#4259)

[28] Siegfried Moran, Dimension subgroups modulo n, Proc. Cambridge Philos. Soc. 68 (1970), 579-582, DOI 10.1017/s0305004100076556. MR0267003

[29] Stefan Papadima and Alexander I. Suciu, Chen Lie algebras, Int. Math. Res. Not. 21 (2004), 1057-1086, DOI 10.1155/S1073792804132017. MR2037049

[30] _ Algebraic invariants for right-angled Artin groups, Math. Ann. 334 (2006), no. 3, 533-555, DOI 10.1007/s00208-005-0704-9. MR2207874

[31] Stewart B. Priddy, Koszul resolutions, Trans. Amer. Math. Soc. 152 (1970), 39-60, DOI 10.2307/1995637. MR0265437

[32] Daniel G. Quillen, On the associated graded ring of a group ring, J. Algebra 10 (1968), 411-418, DOI 10.1016/0021-8693(68)90069-0. MR0231919 
[33] , Rational homotopy theory, Ann. of Math. (2) 90 (1969), 205-295, DOI 10.2307/1970725. MR0258031

[34] Henrika Maria Runde, The lower p-central series of RAAGs, Bachelor thesis, Universität Göttingen, 2018.

[35] William Schmitt, Hopf algebras and identities in free partially commutative monoids, Theoretical Computer Science 73 (1990), 335-340, DOI https://doi.org/10.1016/03043975(90)90184-J.

[36] Jean-Pierre Serre, Lie algebras and Lie groups, Lecture Notes in Mathematics, vol. 1500, Springer-Verlag, Berlin, 2006. 1964 lectures given at Harvard University; Corrected fifth printing of the second (1992) edition. MR2179691

[37] _ Algèbre locale. Multiplicités, Cours au Collège de France, 1957-1958, rédigé par Pierre Gabriel. Seconde édition, 1965. Lecture Notes in Mathematics, vol. 11, Springer-Verlag, Berlin-New York, 1965 (French). MR0201468

[38] Brad Shelton and Sergey Yuzvinsky, Koszul algebras from graphs and hyperplane arrangements, J. London Math. Soc. (2) 56 (1997), no. 3, 477-490, DOI 10.1112/S0024610797005553. MR1610447

[39] Dennis Sullivan, Infinitesimal computations in topology, Inst. Hautes Études Sci. Publ. Math. 47 (1977), 269-331 (1978). MR0646078

[40] Moss E. Sweedler, Hopf algebras, Mathematics Lecture Note Series, W. A. Benjamin, Inc., New York, 1969. MR0252485

[41] Richard D. Wade, The lower central series of a right-angled Artin group, Enseign. Math. 61 (2015), no. 3-4, 343-371, DOI 10.4171/LEM/61-3/4-4. MR3539842

[42] Hans Zassenhaus, Ein Verfahren, jeder endlichen p-Gruppe einen Lie-Ring mit der Charakteristik $p$ zuzuordnen, Abh. Math. Sem. Univ. Hamburg 13 (1939), no. 1, 200-207, DOI 10.1007/BF02940757 (German). MR3069703

\section{ACKNOWLEDGMENTS}

We are deeply grateful to Jacques Darné, Pierre de la Harpe and the anonymous referee for their well-thought comments on preliminary versions of our text.

E-mail address, L.B.: laurent.bartholdi@math.uni-goettingen.de

E-mail address, H.H.: henrikamaria.runde@stud.uni-goettingen.de

E-mail address, T.S.: thomas.schick@math.uni-goettingen.de

(L.B., T.S., H.R.) Mathematisches Institut, Universität Göttingen, Germany

(L.B.) École Normale Supérieure, Lyon, France 